\title{
Stigma's Uneven Decline
}

\author{
Rachel Kahn Best \\ University of Michigan \\ rkb@umich.edu \\ Alina Arseniev-Koehler \\ University of California, Los Angeles \\ arsena@ucla.edu
}

Copyright 2022, Rachel Kahn Best and Alina Arseniev-Koehler. All rights reserved.

\begin{abstract}
Has disease stigma declined? Our ability to answer this question has been hampered by the lack of comparable data across diseases and over time. Using word embeddings, we analyze 4.5 million news articles to create new measures of stigma for 107 health conditions. We find that in the 1980s, most diseases were marked by strong connotations of disgust, danger, impurity, and negative personality traits. Since then, stigma has declined dramatically for most physical illnesses; cancers, neurological conditions, genetic diseases, and many other conditions have shed most of their negative connotations. But this decline was uneven: mental illnesses, eating disorders, and addictions saw no stigma declines, and stigma declined more slowly for infectious diseases than for chronic conditions. Using multivariate regression, we find that patients' activism explains some but not all of the variation in stigma. Stigma has transformed from a sea of negative connotations surrounding most diseases to a narrower set of judgments targeting conditions where the primary symptoms are aberrant behaviors.
\end{abstract}


Since the mid-twentieth century, social movements have fought back against the stigma targeting many diseases, and major cultural changes have reshaped how Americans think about health issues. These dramatic social changes led some scholars to ask whether we have seen the "end of stigma" (Green 2009). Stigma researchers responded skeptically, but due to the expense of collecting representative survey data, we have virtually no comparable measures for hundreds of other potentially stigmatized conditions. Has our society become more tolerant and inclusive for people with diseases and disabilities? If so, has the improvement been equal for all diseases?

To answer these questions, we need to develop comparable measures of stigma that may be assessed across diseases and over time. This paper uses computational methods to analyze the traces stigma leaves in written language, yielding measures of the stigma targeting 107 conditions. We analyze approximately 4.7 million news articles from 1980 through 2018 using word embeddings, a machine-learning text analysis framework that allows us to position diseases according to their connotations of disgust, danger, impurity, and negative personality traits. Using these new measures of stigma, we first ask, has stigma towards diseases declined? Second, did all diseases follow the same trajectory? Third, what explains why stigma declined for some diseases and not others? Fourth, as a result of these changes, which diseases are the most stigmatized, and why?

We find that stigma has indeed declined for most diseases. But these declines were uneven: mental illnesses, eating disorders, and addictions retain as many negative connotations as they had in the 1980s. Moreover, infectious diseases saw smaller stigma declines than chronic conditions. Multivariate analyses reveal that changes in stigma cannot be explained by changes in medicalization or decreased reticence about public discussions of illness. Patients' activism explains some of the declines: disease advocacy expanded over time, and diseases targeted by 
more nonprofits and lobbying tend to be less stigmatized. But while disease-specific advocacy may have contributed to some disease-specific stigma declines, we find more evidence of a broader cultural change that cut across disease categories. In the past four decades, stigma has transformed from a sea of negative connotations surrounding most diseases to a targeted set of judgments about a few disfavored conditions: those in which the primary symptoms are aberrant behaviors.

\section{Defining Disease Stigma}

Goffman defined stigma as an "attribute that is deeply discrediting" that leaves the bearer "tainted" and "discounted" (Goffman 1963:3). More recent scholars have elaborated a multi-step process in which 1) differences between people are labeled; 2) these labels are associated with "negative attributions or stereotypes;" 3) people distance themselves from stigmatized individuals, who then 4) experience "status loss and discrimination" (Pescosolido and Martin 2015:92; see also Link and Phelan 2001). Our study focuses on the second step in this processthe negative attributions or stereotypes associated with various diseases. We focus on several specific negative attributions suggested by prior literature on disease stigma and the construction of disease (e.g., Jones et al. 1984). Our data on these cultural constructions will allow future studies of their effects on other stages of the stigma process, including social distancing, status loss, and discrimination.

In theory, any human difference can be subject to stigma. Researchers have studied stigmas of everything from neighborhoods (Sampson and Raudenbush 2004) to poverty (Reutter et al. 2009) to voluntary childlessness (Park 2002) to sexual orientation (Herek 2004). But most sociological stigma research has focused on health conditions such as mental illness, HIV/AIDS, 
epilepsy, and cancer (Pescosolido and Martin 2015). Just as health issues are central to stigma research, stigma is a central concept for health research. A main focus for medical sociologists is the social construction of illness, which includes a focus on stigma as one of the cultural meanings surrounding illnesses (Conrad and Barker 2010:S69). While recognizing the broad reach of the stigma concept, we focus here on the measurement of disease stigma in particular.

\section{Has Disease Stigma Declined?}

Stigma has long constrained the lives of millions of people with serious diseases. In the mid-twentieth century, people with mental illnesses were often feared, rejected, and/or subjected to involuntary confinement (Phelan et al. 2000; Star 1952). People with epilepsy could be forbidden to marry, barred from employment, and subject to forced sterilization (Schneider and Conrad 1980). In the 1970s, people with cancer were often feared and shunned (Sontag 1990). In the 1980s, calls to identify, confine, or even tattoo people with HIV aroused little public outcry (Arno and Feiden 1992; Chambré 2006; Epstein 1996; Kayal 1993; Brier 2009; Gould 2009).

Stigma's deadly consequences for life and liberty fueled powerful social movements. People with mental health conditions and disabilities came together to demand better treatments; cancer patients disclosed their diagnoses and launched activist movements; healthcare consumers demanded more autonomy in their interactions with physicians; and patients suffering from hundreds of diseases founded nonprofits, organized voluntary associations, and lobbied the government (Anspach 1979; Bastian 1998; Carpenter 2010:337-41, 411-15; Mintzes and Hodgkin 1996; Morgen 2002; Ruzek 1979; Shapiro 1993; Tomes 2006). Many of these advocates explicitly worked to reduce the stigma of their conditions. 
In addition to patients' activism, broader social changes may also have reduced disease stigma. Some scholars have argued that Western societies have become more tolerant of outgroups (Janmaat and Keating 2019; Sullivan 2004). For instance, Charlesworth and Banaji (2019) find that from 2007 to 2016, Americans' attitudes around sexual orientation, race, and skin tone moved towards neutrality. Shifting perceptions of the causes of health conditions may also have reduced disease stigma. Health conditions were increasingly perceived as medical problems instead of moral failings, and increasingly attributed to genetic causes (Brown 1995; Conrad 2005; Conrad and Schneider 1980). Some theorists have suggested that the medicalization and geneticization of illness would lower stigma (Brown 1995; Conrad 2005; Conrad and Schneider 1980), though empirical evidence has been mixed (Dwyer 1992:251; Gollust, Lantz, and Ubel 2010; Kempner 2014; Payton and Thoits 2011; Phelan 2005). (In contrast, other social theorists have argued medicalization itself often includes moral judgments (Zola 1972), and that rather than a decline in stigma, we have seen increased moralization of health (Giddens 1991; Turner 1992).)

These dramatic social changes led some scholars to ask whether we have seen the "end of stigma" (Green 2009). These claims began in the 1980s, with arguments that stigma had ceased "to pose a severe problem" (Gove 1982:290) and that people with mental illnesses had begun to "enjoy nearly total acceptance in all but the most intimate relationships" (Crocetti 1974:88; see discussion in Phelan and Link 1998:S7). Stigma researchers responded skeptically, noting that people with stigmatized diseases still suffer psychological and physiological harms, avoid diagnosis and treatment, lose relationships and jobs, and may even face a shortened life expectancy, in addition to their diseases' physical effects (Link and Phelan 2001; Major and O’Brien 2005; Pescosolido and Martin 2015). But it is challenging for stigma researchers to 
marshal empirical data to reveal whether stigma against diseases has changed over time, whether stigma has changed in the same ways across the vast array of diseases, or whether factors like patient advocacy and medicalization can explain any changes in disease stigma.

\section{The Challenges of Measuring Stigma}

\section{Challenge \#1: Interview and Survey Measures of Stigma are Resource-Intensive}

To see whether stigma has declined over time and whether some diseases are more stigmatized than others, we need comparable data across conditions and over time. However, it is challenging and expensive to collect such data using existing approaches to measuring stigma. Stigma researchers typically employ surveys and interviews to measure the extent to which particular conditions are stigmatized (Link et al. 2004:515). Collecting these self-report data is time- and resource-intensive, making it impractical for any given study to compare large numbers of conditions. Self-report data are also limited in their ability to study changes over time, since contemporary researchers' access to data on past time periods is limited by respondents' memories. It may not be surprising, then, that most studies have collected data at a single point in time on a single condition, or at most a small handful of conditions (but see Pachankis et al. 2018). ${ }^{1}$

For a small number of conditions, researchers have painstakingly collected comparable quantitative data over time. They have found, for instance, that the 1990s saw dramatic declines in some measures of AIDS stigma (Herek, Capitanio, and Widaman 2002). Other studies show

\footnotetext{
${ }^{1}$ In an important exception to the rule, Pachankis et al. (2018) surveyed both experts and the general public about stigma relating to 93 conditions, including approximately 30 distinct health conditions. This is an important step towards comparable data, but still reflects the limitations of survey research: it is expensive, limited to one point in time, and still includes relatively few diseases. A few other studies compare several diseases (Corrigan, Kuwabara, and O’Shaughnessy 2009; Greene and Banerjee 2006; Link et al. 2008; Mak et al. 2006; Marlow, Waller, and Wardle 2015; Pescosolido et al. 2010; Roeloffs et al. 2003; Weiner, Perry, and Magnusson 1988)
} 
that in contrast, mental illness stigma remained remarkably stable. Survey respondents in 1996 were more likely to associate mental illness with violence than they had been in 1950 (Phelan et al. 2000; Phelan and Link 1998). There was no decline in stigmatizing views about schizophrenia, depression, or alcohol dependence between 1996 and 2006 (Payton and Thoits 2011; Pescosolido et al. 2010). These studies were possible because researchers carefully replicated earlier survey questions with similar populations and identical question wording. Due to the expense of collecting representative survey data, we lack data across longer time periods for mental illness and AIDS stigma, and we have virtually no comparable measures for the hundreds of other potentially stigmatized conditions. As Phelan (2005:308) notes, "although stigma is regularly described by scholars as being historically specific, it is just as regularly studied in static social circumstances." Similarly, qualitative work has shown changes in the way we think about particular diseases, particularly cancer (Aronowitz 1998; Clair, Daniel, and Lamont 2016; Lerner 2001; Mukherjee 2010). But these in-depth qualitative studies of particular conditions do not lend themselves to clear comparisons across multiple diseases.

Self-report data make it infeasible for any one study to compare the stigma targeting many diseases over time. But can we create these comparative data by aggregating information across various studies of stigma? Unsurprisingly, definitions of stigma vary across research contexts, disciplinary boundaries, and theoretical orientations (Link and Phelan 2001:365). The widely varying operationalizations of stigma make it difficult for findings to accumulate across studies and rule out direct quantitative comparisons of the levels of stigma measured by various researchers (Pescosolido and Martin 2015:96). Given that combining prior empirical work on stigma is impractical, to compare stigma across diseases and over time we need to develop new measures that are scalable and standardized. 


\section{Challenge \#2: Few Studies Directly Measure Public Stigma}

It can also be challenging to use surveys and interviews to measure broadly shared cultural constructions. Self-report data measure stigma at the individual level, collecting specific respondents' reports of stigmatizing beliefs or behaviors. These methods are ideal for research questions about individual behavior-for instance, when we ask whether people who hold stigmatizing attitudes are more likely to discriminate, we should measure these attitudes and behaviors at the individual level. Survey and interview data yield unmatched insights into perceived stigma (respondents' perceptions of the level of stigma in the population), endorsed stigma (the extent to which respondents embrace stigmatizing attitudes), and anticipated and received stigma (the extent to which stigmatized people expect or have experienced negative interactions) (Pescosolido and Martin 2015).

But other important research questions concern macro-level stigma. For several decades, scholars have criticized stigma research for overemphasizing micro-level feelings and interactions and underemphasizing macro-level social processes of discrimination and exclusion (Hatzenbuehler, Phelan, and Link 2013; Link et al. 2004:512; Link and Phelan 2001:366; Oliver 1990; Sayce 1998). Theorists now recognize that many of stigma's most important consequences involve organizations, institutions, and public policies (Link and Phelan 2001; Parker and Aggleton 2003; Pescosolido and Martin 2015; Sayce 1998). For instance, the stigma associated with schizophrenia could lead to less research funding and fewer professionals who specialize in treating the condition, creating disadvantages for individuals with schizophrenia even if they never experience any negative social interactions due to their illness (Link and Phelan 2001:37273; see also Pescosolido and Martin 2015:96). These approaches conceptualize stigma as 
something that shapes not only individual responses to a diseased person, but also institutional structures that distribute resources to groups of people.

For these macro-level research questions - for instance, questions about how stigma shapes policy, or how activism shapes stigma—scientists would ideally use macro-level measures of shared cultural constructions, or, public stigma. ${ }^{2}$ Yet, these data are rarely collected. For instance, studies of the effectiveness of anti-stigma campaigns are often conducted at the individual level, seeing if individual participants endorse less stigmatizing attitudes after being exposed to campaign materials, rather than measuring change at the societal level (Clair et al. 2016:223; Estroff, Penn, and Toporek 2004; Pescosolido and Martin 2015:105; Stuart, ArboledaFlórez, and Sartorius 2012). ${ }^{3}$

Researchers sometimes operationalize public stigma by aggregating up individual-level measures (e.g., Hatzenbuehler et al. 2014:33). ${ }^{4}$ But collectively shared stigma may be different than the average level of stigma espoused by a representative sample of the population. Cultural theorists recognize differences between "public culture," which is "externalized in the form of symbols, discourses, and institutions," and "personal culture" at the level of the individual

\footnotetext{
${ }^{2}$ This is variously called "public stigma," "community stigma," or "cultural stigma," defined as "the contextual climate of prejudice and discrimination in time and place" (Pescosolido and Martin 2015:94).

${ }^{3}$ Studies of "structural stigma" go beyond the individual level, but they still do not measure collective cultural meanings. Structural stigma has been defined as "societal-level conditions, cultural norms, and institutional policies that constrain the opportunities, resources, and wellbeing of the stigmatized" (Hatzenbuehler and Link 2014:2; see also Clair, Daniel, and Lamont 2016). Some scholars propose correlating stigmatized identities with health outcomes, and identifying structural stigma if, for example, black people or LGBT people have worse health (Hatzenbuehler, Phelan, and Link 2013). But multiple pathways can lead to health disparities, including economic inequality, behavioral patterns, and discriminatory policies; this approach cannot capture the influence of cultural constructions on health. Other scholars identify instances of exclusion and define them as structural stigma. For instance, Lukachko et al. (2014) use what they call "structural measures of racism," including disparities in political participation and incarceration. Corrigan et al. measure structural stigma by identifying "laws that diminish the opportunities of people with mental illness" (Corrigan, Watson, Heyrman, et al. 2005). These studies measure concrete outcomes that might be the result of collective cultural constructions, but they do not attempt to measure those constructions; essentially, they skip over the challenge of measuring collective culture. If we want to provide empirical evidence for the claim that negative attributions, emotions, prejudice, and perceptions of dangerousness have concrete effects, we need to measure these cultural constructions directly.

${ }^{4}$ This article has been retracted due to an error in the data.
} 
(Lizardo 2017:93; see also Eliasoph and Lichterman 2003; Mohr et al. 2020; O. Patterson 2014; Strauss and Quinn 1997; Swidler 2013). Aggregated individual sentiments can be a poor measure of public culture. ${ }^{5}$ For example, the Middle Ages was collectively Christian by its "dominant public culture or collective rules," even if a lot of individual practices were pagan; aggregating individual religious beliefs would underestimate dominance of Christianity in collective culture (Jepperson and Swidler 1994:365-67). The same problem applies if we try to aggregate individual survey responses to measure the collective cultural constructions of various diseases.

One important reason that macro-level stigma may diverge from aggregates of individual-level stigma is that not everyone's ideas are equally influential. Modern definitions of stigma emphasize the importance of power in determining whose ideas shape public stigma, but these theoretical advances have not sufficiently informed how we measure stigma. For example, patients receiving inpatient psychiatric treatment might label staff "pill pushers" and stereotype them as arrogant, but "the patients simply do not possess the social, cultural, economic, and political power to imbue their cognitions about staff with serious discriminatory consequences" (Link and Phelan 2001:376). For Link and Phelan, "what matters is whose cognitions prevailwhose cognitions carry sufficient clout in social, cultural, economic, and political spheres to lead to important consequences for the group that has been labeled as different" (Link and Phelan 2001:378). Similarly, Pescosolido and Martin note that "stigmatization is dependent on core concerns of the sociological enterprise - power and the ability to stratify tangible and intangible resources" (Pescosolido and Martin 2015:91). But how do we measure whose stereotypes "the culture recognizes and deeply accepts" (Link and Phelan 2001:376)? Rather than beginning with individual-level data and attempting to weight respondents by their level of cultural influence,

\footnotetext{
${ }^{5}$ Other critics argue that collective meanings cannot be understood without also measuring individuals' subjective meanings (see discussion in Evans 2009:245).
} 
we turn to text as a direct measure of shared cultural constructions. As we will show, text data offer ways to address each of the three challenges of measuring stigma.

\section{Measuring Public Stigma Using Text Data}

Text-based data can never replace self-report data's unique insights into individuals' beliefs and attitudes. But text has key advantages that offer a path around the roadblocks described above. First, in contrast to surveys and interviews, studying text allows us to affordably compare many conditions and time periods. Second, text allows us to measure public stigma directly, rather than aggregating the attitudes of individuals with varying power to influence collective constructions.

Previous researchers have measured stigma in written language, from newspapers to twitter to medical records. But like other stigma researchers, they usually focus on one or a few conditions at a single point in time (Caspermeyer et al. 2006; Chen and Sokolova 2018; Corrigan, Watson, Gracia, et al. 2005; Dietrich et al. 2006; Flint, Hudson, and Lavallee 2016; Gollust and Lantz 2009; Heuer, McClure, and Puhl 2011; Joseph et al. 2015; Kelly, Saitz, and Wakeman 2016; Kool et al. 2013; Looper and Kirmayer 2004; Reavley and Pilkington 2014; Robinson et al. 2019; Vahabzadeh, Wittenauer, and Carr 2011). Historical studies also look to text for stigma, but do not generally quantify the data (Clair et al. 2016; Wailoo 2006).

Developing stigma measures for dozens of diseases over decades requires analyzing an enormous amount of text. We want to pick up relatively subtle stigmatizing connotations, not just explicit pronouncements that people with a particular condition are bad or immoral. Stigma may sometimes be this explicit; for instance, news outlets in the early 1980s may have published demonizing and dismissive depictions of people with AIDS. But we also want to pick up subtler 
forms of stigma - the differences, for example, between how lung cancer and breast cancer patients are described today. These descriptions will vary substantially from one text to the next. Presumably, people with lung cancer and breast cancer are both often described positively and negatively. We would not expect every description of lung cancer to include moralistic language that blames smokers for getting cancer. But we would expect more of these moralizing discussions of lung cancer than breast cancer. An interpretive analysis of a small number of texts could not identify changes in the probability that a disease will be discussed in a stigmatizing way.

To pick up subtle differences that do not appear in every single text, and to do so for many different diseases, we need to analyze a very large amount of text. The amount of text required makes it infeasible to use human coding, both because of the amount of time that would be required and the difficulty of reliably coding subtle differences across large numbers of texts. The "high noise-to-signal ratio" suggests that formal modeling may be superior to a more interpretive analysis (Lee and Martin 2015:10). At the same time, our goals do not require an indepth understanding of the details of any particular author's argument. To analyze the large numbers of texts required, we turn to computational text analysis.

\section{Word Embedding Methods for Computational Text Analysis}

Most types of computational text analysis that have been adopted by sociologists are illsuited to measure the stigma targeting diseases. Topic models (DiMaggio, Nag, and Blei 2013; Mohr and Bogdanov 2013; Nelson 2017) are better suited to studying which themes arise in particular texts than to understanding the connotations of particular words. Other models focus on counting the numbers of words in texts or mapping the frequency with which words appear 
near each other (Carley 1994; Hoffman et al. 2018; Lee and Martin 2015; Pachucki and Breiger 2010). However, even closely related words might rarely appear near each other in text (Kozlowski, Taddy, and Evans 2019:910). For instance, herpes and obesity rarely appear near each other, but they tend to appear in similar contexts related to impurity. Counting and mapping the co-occurrence of words cannot pick up these types of associations as effectively (Kozlowski et al. 2019; Spillman 2015).

To solve these problems, we turn to word embeddings using Word2Vec, an algorithm that represents each word in the vocabulary as an $\mathrm{N}$-dimensional vector (a list of $\mathrm{N}$ numbers, with $\mathrm{N}$ usually between 100 and 500), based on how the word is used in a text dataset (Mikolov, Chen, et al. 2013; Mikolov, Sutskever, et al. 2013; Rong 2014). ${ }^{6}$ A vector may be imagined as a point in space; for example, a 2-dimensional vector locates a point with an $\mathrm{X}$ and a $\mathrm{Y}$ coordinate on a plane. Words that occur in more similar linguistic contexts have more similar vectors and are therefore closer together, as measured by cosine similarity, the cosine of the angle between the two vectors. For example, we would expect 'woman' and 'man' to hold similar quantitative representations because they are used to describe similar concepts—-both are singular nouns referring to human adults.

Word embeddings have recently been used to measure cultural representations, with impressive results (e.g., Arseniev-Koehler and Foster 2020; Boutyline, Arseniev-Koehler, and Cornell 2020; Caliskan, Bryson, and Narayanan 2017; Charlesworth et al. 2021; Garg et al. 2018; Joseph and Morgan 2020; Kozlowski et al. 2019; Nelson 2021). Caliskan et al. (2017) and Garg et al. (2018) used word embeddings to identify stereotypes related to race, ethnicity, and gender. Kozlowski et al. (2019) traced historical understandings of social class. These studies

\footnotetext{
${ }^{6}$ To create these word-vectors, the algorithm is iteratively fed short excerpts of the corpus as examples to learn from. Each excerpt consists of a target word and the $\mathrm{C}$ words before and after the target word (the context words). The parameter $\mathrm{C}$ is set by the researcher, and usually ranges between 2 and 10 .
} 
provide empirical validation that word embeddings can capture shared cultural representations.

Word2Vec not only learns the meaning of particular words, but reveals relational concepts, such as gender, as lines between these points (Arseniev-Koehler and Foster 2020; Kozlowski et al. 2019). For example, gender may be represented in semantic space as a line ranging from points in space representing femininity (e.g., word-vectors for "feminine" and "woman") to those representing masculinity (e.g., word-vectors for "masculine" and "man.”) masculine. Thus, in addition to describing the location of a particular disease - seeing what words appear near hepatitis, and how they are similar to and different from the words that appear near breast cancer — we can identify key dimensions of the discursive space, identifying axes of judgment and exploring how they intersect.

\section{Operationalizing Stigmatizing Meanings in Text}

Drawing on existing theories, we focus on four dimensions of stigma, each linked to a negative attribution or stereotype: disgust, danger, impurity, and negative personality traits. ${ }^{7}$ Our first stigma dimension is disgust - the extent to which a condition is perceived as "repellant, ugly, or upsetting" (Jones et al. 1984:24). ${ }^{8}$ Our focus on disgust resonates with the stigma' literature's focus on negative emotions (Jones et al. 1984; Link et al. 2004:513). Reactions of disgust may be immediate and difficult to control, possibly making them resistant to stigmareduction efforts (Jones et al. 1984:50). ${ }^{9}$

\footnotetext{
${ }^{7}$ Three of our four stigma dimensions have analogues in Jones et al.'s (1984) synthesis of the key dimensions used by earlier stigma researchers.

${ }^{8}$ Jones et al. called this dimension aesthetics.

${ }^{9}$ Some researchers argue that revulsion toward signs of infectious diseases is an evolved response, biologically rather than culturally determined (Jones et al. 1984:24; Pachankis et al. 2018:453). However, differing cultural responses to conditions like obesity show that much of what people find disgusting is culturally specific (Jones et al. 1984:51). Our analysis brackets the question of whether some diseases are "naturally" more disgusting than others; we focus on the extent to which diseases are depicted as disgusting, regardless of the source.
} 
Our second dimension is danger. Fear and threat are sometimes described as the most important components of stigma (Jones et al. 1984:65), and perceptions of dangerousness have been identified as a key component of mental illness stigma (Link et al. 1999; Pescosolido 2013). Perceived danger can involve both physical and social threats; "even the capacity to be unpredictable, erratic, or irrational can be highly threatening when possessed by persons who are in, or may gain, positions of high social power" (Jones et al. 1984:68). It can also involve the fear of contagion, which can be experienced even for diseases that are not actually infectious. For instance, people may avoid contact with those with cancer or physical disabilities out of a superstitious or emotional fear of contagion (Jones et al. 1984:69; Sontag 1990:6, 58).

Third, we consider impurity, the extent to which a condition is viewed as polluting, contaminating, or immoral. Researchers going back to Goffman (1963) have argued that stigmas are viewed as moral defects. ${ }^{10}$ Some conditions are viewed as immoral because patients are believed to be responsible for their own illness; an extensive research literature finds that people respond more negatively when they believe patients are to blame for their condition (Corrigan et al. 2000; Lebel and Devins 2008; Link et al. 2004:521; Rush 1998; Sontag 1990:57, 100, 144; Weiner, Perry, and Magnusson 1988). Other conditions call up their own moral judgments; for instance, people with depression may be perceived as lazy and self-indulgent (Link et al. 1999; Wood et al. 2014). But even people with "physical deformities, leprosy, sensory deficits, even mental disorders" may be viewed as "morally denigrated and tainted," perhaps because observers wish to believe that such conditions only befall the undeserving (Jones et al. 1984:88).

Fourth, we measure the extent to which diseases are associated with negative personality traits, as opposed to positive personality traits. Theories of stigma hold a prominent place for

\footnotetext{
${ }^{10}$ Immorality plays a surprisingly small role in Jones et al.'s dimensions, despite their recognition that stigmas are often viewed "as signs of moral defect" (Jones et al. 1984:88). Their origin dimension touches on morality by including whether people are blamed for their condition.
} 
"negative attributions or stereotypes" (Pescosolido and Martin 2015:91); when a disease is more strongly linked to negative personality traits than positive ones, we view this as a sign that it is marked by these attributions. To observe these dimensions empirically, we collected and analyzed news media texts.

\section{Methods}

\section{Sampling Text Data}

We track the stigmatizing meanings of diseases across time in U.S. news media. Media texts have the advantage of being archived across decades (as opposed to, for example, Twitter data). They represent relatively elite discourse. We first selected media sources and then sampled texts within each source. ${ }^{11}$ We sought a wide range of American news media outlets in various parts of the country with varying ideological leanings, excluding fringe outlets with low circulations. We collected data through LexisNexis, which archives many of top media outlets and offers bulk downloading through an API to researchers from subscribing institutions. We first selected newspapers that were both available in the Lexis API and appeared on one of several lists of the top 50 newspapers by circulation (infoplease.com 2007; Wikipedia 2019; Yahoo Finance 2017). This yielded a list of seventeen newspapers, including papers in all regions of the country and three of the top five papers (USA Today, the New York Times, and the New York Post). We also included two major newswires (Reuters and the Associated Press) and two mainstream news magazines (U.S. News \& World Report and Newsweek). We included transcripts of news broadcasts from three mainstream broadcast networks (NBC, ABC, and CBS). Finally, we included transcripts from National Public Radio's “All Things Considered”

\footnotetext{
${ }^{11}$ We chose to create our own text corpus, rather than using a pre-existing one (e.g., Google News), so that we would have control over which sources were included and how the data was processed and modeled.
} 
and two partisan TV networks (Fox News and MSNBC), for a total of twenty-seven news

sources (see Table 1).

\section{TABLE 1 ABOUT HERE}

We then used the Lexis Nexis API to collect all news articles published by these outlets that either referred to one of our diseases and/or included any of a broad list of health-related words. ${ }^{12}$ This process yielded a total of 4,711,524 articles from 1980 to 2018 (inclusive). To analyze this data across time, we divided this articles up in three-year periods, beginning with 1980-1982 (inclusive). We selected periods of three years to maximize granularity of time points while also ensuring sufficient data in each period to train high quality word embeddings. The number of articles per time period ranged from 92,494 (for 1983-1985) to 660,747 (2001-2003).

\section{Word2Vec Models}

We took several steps to clean the data. First, we combined multiple names for each disease into a single reconciled term. ${ }^{13}$ Second, we disambiguated terms with multiple meanings - for example, we distinguished between references to depression as a mental illness and a financial crisis. Finally, we removed punctuation, lowercased all text, and converted twoand three-word phrases into single terms. Details on text preprocessing are in Appendix 1.

Next, we trained 25 bootstrapped embeddings for each time period using procedures described in Appendix 1. We then defined dimensions for four aspects of stigma: disgust,

\footnotetext{
${ }^{12}$ Our subscription to the Lexis API imposed limits on the numbers of downloads, making it infeasible to download the entire text of each media outlet. Instead, we focused on the most relevant articles for measuring disease stigma. We compiled a list of 166 search terms that included various names for all the diseases of interest; broader terms including disorder, disease, syndrome, illness, and death; and a list of symptoms and aspects of life that are often medicalized including sadness, sleep, and sex. We used regular expressions to search for multiple possible grammatical forms of these terms. These search terms were intentionally broad to capture a wide range of articles while including the most relevant text for mapping disease stigma.

${ }^{13}$ For example, all references to diffuse myofascial pain syndrome, fibromyositis, fibrositis, and FMS were replaced with fibromyalgia.
} 
danger, impurity, and negative personality traits. For each dimension, we selected "anchor words" for each pole of the dimensions-e.g., purity and impurity. We used these anchor words to extract a line corresponding to the dimension in semantic space (e.g., a line from pure to impure). See Appendix 1 for a discussion of how we selected anchor terms and validated our dimensions.

To measure the stigma targeting a disease in a given time period, we computed the cosine similarity between the word-vector for the disease name and the stigma dimension, yielding a "stigma score" ranging from -1.0 to $1.0 .{ }^{14} \mathrm{~A}$ more positive score suggests that the disease lies closer to the stigmatized pole of the dimension (e.g., danger), and a more negative score suggests that the disease lies closer to the non-stigmatized pole of the dimension (e.g., safety). We then standardized each disease's score against the scores for all other vocabulary words in the embedding. The final scores can be interpreted as the number of standard deviations more (or less) stigmatized a word is compared to all other vocabulary words. Finally, we created an overall "stigma index" defined as the mean of the four dimensions.

Beginning with a list of 116 diseases, we excluded 10 diseases that appeared fewer than 20 times in every three year time period. To aid interpretation and visualization of our results, we grouped the 106 remaining diseases into analytical categories (see Table 2). In grouping diseases, we were guided by ICD-10 codes but also by stigma theory—-for example, we grouped together several diseases commonly thought to have causes associated with people's lifestyles, including poor diet, lack of exercise, drinking, and smoking, since these might be expected to be stigmatized if patients are blamed for their conditions. These classifications are imperfect.

\footnotetext{
${ }^{14}$ We use bootstrapping to estimate how each disease scored on each stigma dimension in each time period. For example, to estimate the "danger" of depression in the time period 1980-1982, we computed the "danger" of depression in each of the 25 bootstrapped models, and then computed the $92 \%$ confidence interval for this estimate across these 25 bootstrapped models.
} 
Sometimes the categorization is disputed or scientifically unclear-for example, there is some debate as to whether asthma is an autoimmune condition. Other diseases could fit into more than one category (e.g., muscular dystrophy is both genetic and neurological). These categories form the groups for Figures 1 and 2, and we also use mental illnesses, addictions, and eating disorders as independent variables in our regression models.

\section{TABLE 2 ABOUT HERE}

\section{Validation}

We took several steps to validate our dimensions and the resulting stigma scores. First, cross validation revealed that our dimensions classified the anchor words with a high level of accuracy (e.g., as disgusting versus enticing). Second, we examined the words in the vocabulary that loaded highest on each pole of each dimension, and found that their meanings aligned with our stigma concepts. Third, we validated our stigma measures against scores from Pachankis et al.'s (2018) survey in which stigma experts were asked to rate the stigma targeting several dozen conditions. For each of our four stigma measures, diseases that our models rate as more stigmatized were ranked by experts as receiving significantly more social distance, an outcome often examined in research on stigma (Link and Phelan 2001; Pescosolido and Martin 2015). Additionally, our measures of impurity, disgust, and danger were significantly correlated with parallel rankings by experts. See Appendix 1 for further details on validation.

\section{Independent Variables}

Having created stigma scores for each disease in each time period, we then operationalized several independent variables that might help explain why some diseases are 
more stigmatized than others. Two are derived from the text data: medicalization and word count. The first captures the extent to which a disease is discussed in context of medicine and is operationalized as the cosine similarity between a disease and the average of 16 word-vectors about medicine (e.g., doctor, prescription, pharmacology, medicine, and medication). The second captures the amount of attention to a disease and is a standardized count of the number of times the disease was mentioned in each time period.

Our analyses also include an independent variable noting the level of advocacy targeting each disease in each time period. This variable draws on data collected by Best (2019) on the number of nonprofits targeting a particular disease in a particular year and the lobbying expenditures related to each disease. We collected new data on several conditions not included in Best's dataset (addictions, auditory and visual impairments, and several mental illnesses). We set the nonprofits and lobbying variables to their means for each three-year period, standardized each variable, and created an advocacy index equal to their mean. For details on these data, see Best (2019:174-78). Table 3 presents descriptive statistics for all variables used in the analysis.

\section{TABLE 3 ABOUT HERE}

\section{Statistical Analyses}

We constructed a dataset with one observation for each disease in each three-year time period. So, for instance, one line of data includes schizophrenia's scores for each stigma dimension in the 2012-2015 news texts, how much advocacy targeted schizophrenia in those three years, and how often schizophrenia was referred to in the news in those three years. We exclude lines of data in which the disease name appeared fewer than 20 times in the three-year window of texts. We conducted multivariate linear regressions with the stigma index as the 
dependent variable, with robust cluster standard errors to account for the fact that repeated observations of a given disease in multiple years are not independent (Williams 2000).

\section{Results}

\section{Has Stigma Declined?}

In the 1980s, most diseases in our study were highly stigmatized, marked by an association with disgust, danger, impurity, and negative personality traits. But stigma declined dramatically over time. On the whole, health conditions are dramatically less marked by these negative connotations than they were four decades ago. The thick gray line in Figure 1 shows the mean stigma score over time. ${ }^{15}$ Across all diseases in the study, the mean level of stigma declined by more than 50\%. Model 1 (Table 4) uses a linear time trend to predict the stigma index and confirms that this decline was statistically significant.

\section{FIGURE 1 ABOUT HERE}

\section{TABLE 4 ABOUT HERE}

Breaking down the data into groups of diseases reveals that most disease categories saw stigma declines. For instance, in 1980, cancers, genetic diseases, and neurological conditions were all, on average, about one standard deviation more stigmatized than the average word in the corpus. By 2016, they clustered near zero, no more linked to disgust, danger, impurity, or negative personality traits than the average word in the corpus. Stigma also declined for most other groups of diseases, although infectious diseases, and especially sexually transmitted infections, saw slower declines (see Figure 1).

\footnotetext{
${ }^{15}$ To reveal changes in the way the same diseases are constructed (instead of changes in the mix of diseases appearing in the media), Figure 1 only shows data for the 53 diseases that we observed across all 13 time periods.
} 
However, three types of diseases—-mental illnesses, eating disorders, and addictionsfollowed a dramatically different trajectory. In the 1980s, diseases in these categories were in the upper half of the stigma distribution, somewhat more stigmatized than visual and auditory conditions, genetic conditions, and neurological disorders. But they weren't outliers; many other disease categories were just as stigmatized, including infectious diseases, autoimmune conditions, and arguably even cancers. However, while other disease categories saw dramatic stigma declines, the stigma targeting mental illnesses, eating disorders, and addictions remained stubbornly high across the decades. By the late 1990s, these three types of disorders, and to a lesser extent infectious diseases, stood out for their high levels of stigma relative to other types of diseases (see Figure 1).

Models 2 and 3 (Table 4) show that these divergences over time across disease categories are statistically significant. Model 2 includes the time trend and dummy variables denoting whether a disease was a mental illness, an eating disorder, or an addiction; diseases in these groups are significantly more stigmatized than other conditions. All three coefficients are large: since the dependent variable is a mean of four standardized stigma scores, the fact that the coefficients are all above 0.8 indicates that diseases in these three categories tended to be almost a full standard deviation more stigmatized than other diseases on each stigma dimension. Compared to Model 1, the R-squared increases dramatically, from 0.098 to 0.312 , indicating that whether a disease falls in one of these three categories explains a substantial amount of the variation in stigma. Model 3 adds interaction effects between these three disease categories and time, to test whether the rate of decline across time varies for each of these three disease categories. All three of the year interaction effects are positive, significant, and large enough to cancel out the negative main effect for year. Thus, Model 3 confirms that while most diseases 
saw significant stigma declines, this was not the case for mental illnesses, eating disorders, and addictions.

\section{Explaining Changes in Disease Stigma}

Why did stigma decline for so many diseases? Model 4 (Table 4) adds two variables referring to the amount and type of references to diseases in the media corpus, respectively. First, we include a standardized measure of the number of times the disease appeared in the corpus in a particular year. Absence from mainstream discourse may itself be a marker of stigma. Sontag (1990) evocatively described reluctance to discuss cancer in the 1970s; the implication that the disease was too horrible to mention fostered a sense of shame and dread. Consistent with this observation, we find that diseases that are discussed frequently in the corpus tend to be much less stigmatized than diseases that are rarely mentioned (see Table 4, Model 4).

Model 4 also adds a variable that attempts to measure the extent to which a disease is medicalized. Some scholars have theorized that medicalization might decrease stigma - that thinking of a condition as a medical matter as opposed to a sin or a crime would be associated with lower levels of stigma (Brown 1995; Conrad 2005; Conrad and Schneider 1980). However, other theorists have noted that patients are often blamed for even highly medicalized conditions (Kempner 2014:15; Zola 1972:490-92), and while some research links medicalization to decreases in stigmatization (Gollust et al. 2010), other studies show that medical and genetic understandings of illness either have no effect on stigma or can even backfire (Angermeyer et al. 2011; Dwyer 1992:251; Payton and Thoits 2011; Pescosolido 2013; Pescosolido et al. 2010; Phelan 2005; Read et al. 2006). Most existing research would predict either a negative or a zero coefficient for medicalization. In contrast, we find a significant positive relationship between 
medicalization and stigma: diseases that are discussed in a more medicalized context tend to be more stigmatized than other diseases.

We believe this result stems in part from the fact that all the diseases in our sample are quite medicalized; we are not comparing conditions currently constructed as medical issues to conditions currently constructed as sins or crimes (e.g., adultery or homicide). All the diseases in our sample are likely less stigmatized than behaviors that are primarily understood as sinful or criminal. ${ }^{16}$ Additionally, our medicalization variable may be distinguishing between diseases that are only discussed in medical contexts to those that make it into the news in other ways. While some of the ways a disease might become newsworthy are potentially stigmatizing, as when a criminal defendant's diagnosis of mental illness is mentioned, most are not. Diseases that seem less medicalized in media text may be those that appear in human-interest stories about patients, descriptions of medical research breakthroughs, and requests for donations to non-profits in obituaries. Thus, discussion in non-medicalized contexts could indicate that a disease has been constructed as a philanthropic cause or a sympathetic topic for a human-interest story.

Comparing Model 4 to Model 3, we see that controlling for mentions and medicalization does not substantially change the time trends or the coefficients for mental illnesses, eating disorders, or addictions. Therefore, we cannot attribute the decline of stigma over time, or the increasing disadvantage for mental illnesses, eating disorders, and addictions, to changes in mentions or medicalization.

\footnotetext{
${ }^{16}$ Our diseases have an average stigma score of $0.58(\mathrm{SD}=0.61)$ in the time period beginning in 2016. We can compare this the average stigma score for terms corresponding to stigmatized identities related to crime, derived from from Pachankis et. al. (2018). These terms include: sex_offender, criminal_record, prostitute, drug_dealer, and gang_member. In this same time period, the average stigma score for these terms is $1.08(\mathrm{SD}=0.57)$.
} 
Model 5 asks whether advocacy can explain why some diseases are more stigmatized than others, and why stigma changed over time. The advocacy index takes account of both the number of nonprofits and the lobbying expenditures targeting a disease in a particular year. Its coefficient is large, negative, and statistically significant, indicating that diseases targeted by more advocacy tended to be less stigmatized. Net of the time trend and the three disease categories in the model, a disease that was one standard deviation higher in terms of advocacy tended to be 0.3 standard deviations lower in terms of stigma.

This pattern does not necessarily mean that advocacy caused stigma to decline. High levels of stigma may also depress advocacy, making patients less likely to found nonprofits (Best 2019). And other characteristics of a disease may increase stigma and suppress advocacy—for instance, acute infectious diseases might face more stigma because of the fear of contagion, and might have less advocacy because patients either die or recover before becoming advocates for the disease. It is difficult to tease out the direction of causality, and our data are not granular enough to make a clean observation of whether advocacy or stigma changes first. But the large advocacy coefficient is, at least, consistent with the possibility that advocacy can reduce stigma.

Does controlling for advocacy help explain why stigma declined overall, and why stigma remained high for some categories of diseases? It's possible that some of the decline in disease stigma can be attributed to the rise of disease patients' activism since the 1980s (Best 2019); comparing Models 4 and 5, the year effect decreases by $20 \%$, from -0.062 to -0.05 . But the decline in the coefficient is not statistically significant, and there remains a large and negative decline of stigma over time that is not explained by our measure of advocacy. Moreover, Model 5 still shows a positive main effect and / or interaction effect for mental illnesses, eating disorders, and addictions, suggesting that these diseases' higher levels of stigma cannot be 
explained by lower levels of advocacy. Interestingly, the coefficient for media mentions moves towards zero and loses statistical significance when we control for advocacy, suggesting that the negative coefficient in Model 4 was actually picking up the effects of advocacy on stigma, with more mobilized diseases receiving more media mentions.

These regressions use the disease as the unit of analysis and look for disease-specific effects - for instance, do diseases with larger advocacy campaigns see larger stigma declines? The limited explanatory value of disease-specific variables points to the importance of changes that cut across disease categories. The large and statistically significant decline in stigma that persists in Model 5, after controlling for diseases' levels of advocacy, raises the possibility that the declining stigma of chronic physical illness was a cultural change that crossed many disease categories.

Americans' experience of illness changed dramatically in the second half of the twentieth century. At midcentury, illness was often experienced privately, with patients deferring to physicians to manage their treatment (Freidson 1970). In the ensuing decades, social movements including the health consumer movement, the women's health movement, and movements promoting occupational and environmental health challenged physicians' authority and pushed for patients' control over their bodies and healthcare (Bastian 1998; Mintzes and Hodgkin 1996; Morgen 2002; Rodwin 1994). Disability activists demanded rights and integration (Fleischer and Zames 2001; Shapiro 1993). Culturally, Americans became more willing to challenge the authority of physicians and scientists, and became more knowledgeable and empowered about their health (Bell 2009; Carpenter 2010:325; McKinlay and Marceau 2002:402; Rose 2007:23; Starr 1982). Celebrities began to go public with diagnoses of breast cancer and other illnesses 
(Ferraro 1993; Kedrowski and Sarow 2007). Together, these changes turned chronic diseases from matters solely for doctors' expertise into matters for public discussion.

Previous scholars have discussed the relationship between these cultural changes and stigma one disease at a time, discussing, for instance, the impact of Republican women's disclosures of breast cancer diagnoses as key to decreasing breast cancer stigma (Casamayou 2001:59; Ferraro 1993; Kedrowski and Sarow 2007:54, 67, 91). The fact that stigma declined in parallel for so many physical illnesses suggests that this process didn't need to happen separately for each disease. Instead, multiple cultural changes came together to produce a new openness about physical illness in general.

\section{The Contemporary Landscape of Disease Stigma}

Four decades of transformations in the cultural construction of physical illness have created new disparities between diseases. Most physical illnesses are coded relatively neutrally along our stigma dimensions, with some even having positive connotations. In contrast, diseases where the primary symptoms are aberrant or deviant behaviors remain highly stigmatized.

Figure 2 shows mean stigma scores from 2010-2018 for each disease. ${ }^{17}$ Diseases are grouped into categories and organized from the group with the highest mean stigma score (addictions) to the lowest (neurological conditions). Within groups, individual diseases are sorted from most to least stigmatized.

\section{FIGURE 2 ABOUT HERE}

The bottom half of Figure 2 reflects the neutral to positive cultural constructions of most chronic physical illnesses. In the contemporary period, the least stigmatized categories of

\footnotetext{
${ }^{17}$ Figure 2 includes mean scores for each disease that was observed at least 20 times in each time period in the decade (2010-2012, 2013-2015, and 2016-2018). For space, we omit diseases in the "other" category.
} 
diseases are neurological conditions, cancers, visual and auditory impairments, and genetic disorders. Very few diseases in these categories have stigma scores above 0.5 , reflecting the fact that they are, on average, no more than half a standard deviation more linked to disgust, danger, impurity, or negative personality traits than the average word in the corpus. Several of the diseases in these categories actually have negative scores on the stigma index. This means that on average, diseases like breast cancer, multiple sclerosis, and cystic fibrosis were coded as more pure than impure, more safe than dangerous, more appealing than disgusting, and/or more linked to positive than negative personality traits. Indeed, prior work has suggested that breast cancer survivors are now perceived as "beacons of hope" and signals of courage and vitality (King 2004). While the transformation in the meanings of breast cancer is well-known (e.g., King 2004; Sally E. Thorne, Carol Murray 2000; Sontag 1990), our results additionally suggest that a small handful of other diseases may have undergone similarly radical transformations.

The top of Figure 2 reflects the extreme stigma targeting diseases for which the primary symptoms are aberrant or deviant behaviors. Eating disorders, mental illnesses, and addictions are more stigmatized than any other group of diseases. Their stigma scores are tightly clustered together: None of the twelve diseases in these categories has a stigma score under 1.3, meaning that their scores for danger, disgust, impurity, and negative personality traits are, on average, at least 1.3 standard deviations higher than the average vocabulary word in the corpus. These twelve conditions make up twelve of the fifteen most stigmatized diseases in our study.

Why are eating disorders, addictions, and mental illnesses the most stigmatized disease categories? Some theories might lead us to expect that these conditions are less medicalized than physical illnesses. However, Models 4 and 5 (Table 4) showed that diseases in these categories are still significantly more stigmatized than others, even when controlling for medicalization (at 
least as measured in text). Their key commonality seems to be that the primary symptoms are deviant behaviors. Even when a disease is thought of as a medical issue and/or genetically caused, it may still attract high levels of stigma if patients are thought of as fundamentally different kinds of people (Kempner 2014:163; Link et al. 1999). Thus, the decline in stigma targeting most physical illnesses that we described in the previous section has created a stark distinction between diseases with primarily behavioral symptoms and all other illnesses.

The contemporary stigma landscape also reveals other types of variation between diseases, in line with existing theoretical predictions. Attribution theory suggests that patients are more stigmatized when they are believed to be responsible for their condition (Else-Quest et al. 2009; Jones et al. 1984:57; Lebel and Devins 2008; Marlow, Waller, and Wardle 2015; Rush 1998; Weiner et al. 1988). We observe a stigma gradient across cancers that is consistent with this prediction. We find that among cancers, breast and pancreatic cancers are stigmatized the least, and uterine, liver, cervical, and skin cancers stigmatized the most. The higher levels of stigma for skin, liver, and cervical cancers are likely linked to their perceived controllability (by avoiding sun exposure, drinking, and HPV infection). The relatively high levels of stigma targeting sexually transmitted infections could also be linked to patients' perceived responsibility for their conditions. Syphilis, herpes, gonorrhea, chlamydia, and HPV all have high stigma scores. In addition to perceived controllability, this stigma is also likely linked to moralistic judgments about sexual behavior. HIV/AIDS is an outlier among sexually transmitted infections, with markedly lower stigma scores. ${ }^{18}$ However, despite qualitative evidence of strong links between diet, exercise, and moral judgments (Metzl and Kirkland 2010; Saguy and Riley 2005),

\footnotetext{
${ }^{18}$ We believe that this low score for HIV/AIDS is due to the high level of advocacy, nonprofit activity, and media attention the disease receives, meaning that many references to AIDS in media text refer to galas, fundraisers, etc.we would expect the disease name to have fewer negative connotations than the average reference to herpes or syphilis.
} 
the diseases we classified as having lifestyle-related causes do not seem substantially more stigmatized than musculoskeletal or autoimmune conditions.

Previous researchers have also argued that patients face more stigma when their illnesses are contested by the medical profession, and/or when the primary symptoms are not diagnosable by objective tests (e.g., pain). In line with these predictions, the conditions we identify as contested illnesses (chronic fatigue and immune dysfunction syndrome, fibromyalgia, and inflammatory bowel disease) have relatively high stigma scores as compared to most cancers and genetic conditions. In line with qualitative research (Kempner 2014), migraine is substantially more stigmatized than other neurological conditions, perhaps because the unobservable nature of pain subjects patients to suspicion of malingering.

Because our data allow direct comparisons across diseases, we can put these different sources of stigma in perspective. While blame for controllable conditions and doubt around contested illnesses and pain condition do seem to increase stigma, these processes almost never produce stigma as severe as that targeting mental illnesses, eating disorders, and addictions. The most stigmatizing language is reserved for those conditions in which the symptoms are deviant behaviors, and the patients are viewed as fundamentally different kinds of people.

\section{Discussion}

In The End of Stigma, Green (2009:1) argued that "the old order in which the sick and disabled are disempowered and marginalized is being replaced by a world characterized by their increasing confidence and a reassertion of their essential personhood." Our data dispute Green's optimistic assertion for two of the three disease groups he studied (drug and alcohol abuse and 
mental illness). But his words more accurately describe the changing cultural connotations of most chronic conditions. Our data suggest that a broad swath of diseases have become considerably less shameful, less identity-threatening since the early 1980s. Writing in 1978, Susan Sontag hoped that we could someday "regard cancer as if it were just a disease-a very serious one, but just a disease. Not a curse, not a punishment, not an embarrassment. Without 'meaning." (Sontag 1990:102). While it is unrealistic to expect a disease to be stripped of all cultural meanings (Aronowitz 1998:14), our results reveal that the connotations of many physical illnesses have changed dramatically. Cancers, at least as described in the news media, no longer connote disgust, impurity, or negative personality stereotypes. While cancer may once have been regarded as shameful and discrediting (Sontag 1990), "cancer patient" and "cancer survivor" have arguably become valorized identities. Similar changes have occurred for dozens of other chronic physical illnesses.

We measured declines in the public or cultural stigma linked to many diseases - their shared meanings as reflected in news media text. Future research is needed to determine whether these declines in public stigma led to better outcomes for patients. To understand this possible divergence, consider the fact that over time, Americans have become less willing to espouse explicitly racist attitudes. But the decline in overt racism masks the continued virulence of implicit racism, structural racism, and racist attitudes that are no longer overtly expressed in surveys (Bonilla-Silva 2017; Bonilla-Silva and Dietrich 2011; Rabinowitz et al. 2009; Ray and Seamster 2016). In the race literature, a sophisticated conversation about the implications of these changes over time was made possible by the existence of comparable survey data across decades. Our study attempts to provide that type of evidence base for disease stigma, but should only be the beginning of a conversation about the implications of the patterns we describe-just 
as the survey data are only the beginning for understanding changes in racial attitudes and discrimination.

In contrast to the dramatic declines in stigma for many diseases, the stigma targeting mental illnesses, addictions, and eating disorders has remained remarkably stable. Our data suggest that these conditions continue to connote danger, disgust, impurity, and negative personality traits. Infectious diseases, especially sexually transmitted infections, have also seen slower stigma declines than chronic diseases. Four decades of stigma have created sharp disparities between chronic physical illnesses and conditions where the primary symptoms are aberrant or deviant behaviors.

This divergence between diseases' stigma trajectories was unobservable in the few previous longitudinal studies of stigma. Since most previous researchers have measured stigma using survey data, which are expensive and time-consuming to collect, they have had to focus on small numbers of diseases. In choosing one or a few conditions to study, researchers have usually selected the types of diseases that remain highly stigmatized, such as mental illnesses. These are the conditions that make sense for a contemporary stigma researcher to studysomeone who sets out to measure stigma today would be unlikely to choose a disease like breast cancer or cystic fibrosis. These research decisions, while completely appropriate within the context of individual studies, accumulate into a research literature that has obscured the decline in stigma for most conditions.

In addition to comparing the amount of stigma targeting different diseases and tracing its change over time, future studies using our data can compare the type of stigma that marks different conditions, distinguishing infectious diseases' high levels of disgust from mental illnesses' strong associations with danger and negative personality traits. To reveal changes over 
time more parsimoniously, this paper focused primarily on a single stigma index. However, disaggregating our four stigma dimensions may reveal important differences in the type of stigma that targets different diseases. Future studies can exploit the multidimensionality of our measures to compare the stigma profiles of various diseases. Future work could also explore whether these different types of stigma predict different outcomes for patients, including shame, social distancing, and discrimination. For example, people whose diseases are constructed as impure might face more discrimination, while people with diseases viewed as disgusting or dangerous might face more social distancing.

In addition to its implications for medical sociological treatments of stigma, our study contributes to debates in the sociology of culture on how to measure meaning in text. Cultural sociologists have debated extensively whether interpretive analyses of text, human coding, or formal modeling are better approaches to measure culture in text (Biernacki 2012; Evans 2009; Lee and Martin 2015; Mohr 1998; Reed 2015; Spillman 2015). Our analysis suggests that word embeddings may be particularly useful for comparing cultural constructions across categories and over time, tasks that require analyzing too many texts for interpretive analysis to be feasible. Moreover, in addition to identifying the cultural meanings attached to particular constructs (in this case, diseases), word embeddings also identify the schemas that organize judgments (Arseniev-Koehler and Foster 2020). We identified key dimensions along which the constructions of diseases vary: fear/safety, disgust/attraction, purity/impurity, and positive traits/negative traits. These organizing dimensions are an important aspect of culture, analytically distinct from judgments of particular diseases. For instance, people might disagree about the extent to which a diagnosis of gonorrhea signals immorality, but they might still all tend to rank and understand people, behaviors, and diseases on a scale from moral to immoral. 
Our results also align with calls to directly measure public culture. In a recent book, Mohr et al. argue that "the study of culture and meaning-making centers on how social life is structured by extra-individual forces. And yet... much of our evidential basis for what culture is, and how it operates, rests upon observation of how individuals internalize such processes and change their behaviors because of them" (Mohr et al. 2020). This observation echoes our critique of stigma research. Contemporary theories of stigma recognize the importance of shared collective representations, disproportionately influenced by people with power and therefore distinct from aggregated individual beliefs. This "public culture" of stigma may differ from the "personal culture" of stigmatizing attitudes embraced by individuals (Lizardo 2017:93; see also Eliasoph and Lichterman 2003; Mohr et al. 2020; O. Patterson 2014; Strauss and Quinn 1997; Swidler 2013). Turning to text has the key advantage of directly measuring collective representations, and word embeddings have key advantages in understanding these shared meanings. We used word embeddings to compare the symbolic constructions of various diseases and observe changes in these constructions over time; future researchers could apply these methods to other empirical sites. For instance, word embeddings could provide new comparative data on the moral judgments associated with the beneficiaries of various social policies (Schneider and Ingram 1993; Steensland 2010).

When we turn to text to measure culture, we need to think about which texts to use. For this study, we drew exclusively on news media, a choice that has advantages and disadvantages. News media, as a relatively elite discourse subject to its own professional standards and commercial imperatives, might miss stigmas that would become apparent in other types of text (e.g., twitter posts). On the other hand, news media texts are available across decades, providing insights into how cultural constructions change over time. 
The fact that different types of text reveal different aspects of culture is an argument for strategically selecting text sources, rather than lumping together all available text. For instance, analyzing historical text without ensuring similar representation of genres and subgenres in each time period leaves researchers unable to prove that "any change they observe is not an artefact of differences in genre balance" (Davies 2012:124). Lumping together text genres can also make it harder to interpret the results. For instance, as discussed above, our lower-than-expected stigma for HIV/AIDS can be interpreted by considering how references to this disease make it into the newspaper. Future studies should expand beyond our focus on news media, but should choose text corpuses chosen for theoretical reasons—-for instance, researchers might analyze language in medical records to think about doctors' prejudice, Twitter to think about broader culture, etc.

If one of the advantages of word embeddings is the chance to measure public culture as something distinct from aggregated private culture, we may need new ways to validate word embeddings. Previous studies use aggregate measures of personal culture-e.g., human-rated similarities between words, or humans' implicit associations with words — as the gold standard for validating word embedding models (Caliskan et al. 2017; Kozlowski et al. 2019). But divergences between word embeddings and survey data do not always indicate problems with embedding models. They may also indicate real differences between public and private culture.

Word embeddings have not yet been fully integrated into traditional social science analysis. Most previous social science applications have been primarily descriptive, focused on documenting changes in embeddings over time and validating them against other types of data (Caliskan et al. 2017; Garg et al. 2018; Kozlowski et al. 2019). Our study integrates this new way to measure cultural constructions with traditional social science data on advocacy 
organizations, suggesting ways to combine embeddings with other social science data to answer questions about the determinants and effects of cultural meanings. 


\section{References}

Angermeyer, Matthias C., Anita Holzinger, Mauro G. Carta, and Georg Schomerus. 2011.

"Biogenetic Explanations and Public Acceptance of Mental Illness: Systematic Review of Population Studies." The British Journal of Psychiatry 199(5):367-72. doi: 10.1192/bjp.bp.110.085563.

Anspach, R. R. 1979. "From Stigma to Identity Politics: Political Activism Among the Physically Disabled and Former Mental Patients." Social Science and Medicine 13A:765-73.

Arno, Peter, and Karyn L. Feiden. 1992. Against the Odds: The Story of AIDS Drug Development, Politics And Profits. Harper Collins.

Aronowitz, Robert A. 1998. Making Sense of Illness: Science, Society and Disease. Cambridge University Press.

Arseniev-Koehler, Alina, and Jacob G. Foster. 2020. "Machine Learning as a Model for Cultural Learning: Teaching an Algorithm What It Means to Be Fat." ArXiv Preprint ArXiv:2003.12133.

Bastian, Hilda. 1998. "Speaking up for Ourselves.” International Journal of Technological Assessment in Health Care 14(1):3-23.

Bell, Susan E. 2009. DES Daughters: Embodied Knowledge, and the Transformation of Women's Health Politics in the Late Twentieth Century. Temple University Press.

Best, Rachel Kahn. 2019. Common Enemies: Disease Campaigns in America. New York, NY: Oxford University Press.

Biernacki, Richard. 2012. Reinventing Evidence in Social Inquiry: Decoding Facts and Variables. New York: Palgrave Macmillan.

Bonilla-Silva, Eduardo. 2017. Racism without Racists: Color-Blind Racism and the Persistence of Racial Inequality in America. 5th Edition.

Bonilla-Silva, Eduardo, and David Dietrich. 2011. The New Racism: The Racial Regime Of PostCivil Rights America. Brill.

Boutyline, Andrei, Alina Arseniev-Koehler, and Devin Cornell. 2020. "School, Studying, and Smarts: Gender Stereotypes and Education Across 80 Years of American Print Media, 1930-2009." SocArXiv. doi: 10.31235/osf.io/bukdg.

Brier, Jennifer. 2009. Infectious Ideas: U.S. Political Responses to the AIDS Crisis. Chapel Hill: The University of North Carolina Press.

Brown, Phil. 1995. "Naming and Framing: The Social Construction of Diagnosis and Illness." Journal of Health and Social Behavior 34-52. doi: 10.2307/2626956. 
Caliskan, Aylin, Joanna J. Bryson, and Arvind Narayanan. 2017. "Semantics Derived Automatically from Language Corpora Contain Human-like Biases.” Science 356(6334):183-86.

Carley, Kathleen. 1994. "Extracting Culture through Textual Analysis.” Poetics 22(4):291-312. doi: 10.1016/0304-422X(94)90011-6.

Carpenter, Daniel. 2010. Reputation and Power: Organizational Image and Pharmaceutical Regulation at the FDA. Princeton University Press.

Casamayou, Maureen Hogan. 2001. The Politics of Breast Cancer. Washington, D.C.: Georgetown University Press.

Caspermeyer, Joseph J., Edward J. Sylvester, Joseph F. Drazkowski, George L. Watson, and Joseph I. Sirven. 2006. "Evaluation of Stigmatizing Language and Medical Errors in Neurology Coverage by US Newspapers.” Pp. 300-306 in Mayo Clinic Proceedings. Vol. 81. Elsevier.

Chambré, Susan Maizel. 2006. Fighting for Our Lives: New York's AIDS Community and the Politics of Disease. Rutgers University Press.

Charlesworth, Tessa E. S., and Mahzarin R. Banaji. 2019. "Patterns of Implicit and Explicit Attitudes: I. Long-Term Change and Stability From 2007 to 2016.” Psychological Science 30(2):174-92. doi: 10.1177/0956797618813087.

Charlesworth, Tessa ES, Victor Yang, Thomas C. Mann, Benedek Kurdi, and Mahzarin R. Banaji. 2021. "Gender Stereotypes in Natural Language: Word Embeddings Show Robust Consistency across Child and Adult Language Corpora of More than 65 Million Words." Psychological Science 32(2):218-40.

Chen, Qufei, and Marina Sokolova. 2018. "Word2Vec and Doc2Vec in Unsupervised Sentiment Analysis of Clinical Discharge Summaries.” ArXiv:1805.00352 [Cs].

Clair, Matthew, Caitlin Daniel, and Michèle Lamont. 2016. "Destigmatization and Health: Cultural Constructions and the Long-Term Reduction of Stigma." Social Science \& Medicine 165:223-32.

Conrad, P., and J. W. Schneider. 1980. Deviance and Medicalization: From Badness to Sickness. St. Louis, MO: The C.V. Mosby Company.

Conrad, Peter. 2005. "The Shifting Engines of Medicalization.” Journal of Health and Social Behavior 46(1):3-14. doi: 10.1177/002214650504600102.

Conrad, Peter, and Kristin K. Barker. 2010. "The Social Construction of Illness: Key Insights and Policy Implications.” Journal of Health and Social Behavior 51(1_suppl):S67-79. doi: 10.1177/0022146510383495. 
Corrigan, Patrick W., Sachiko A. Kuwabara, and John O’Shaughnessy. 2009. “The Public Stigma of Mental Illness and Drug Addiction: Findings from a Stratified Random Sample." Journal of Social Work 9(2):139-47.

Corrigan, Patrick W., L. Philip River, Robert K. Lundin, Kyle Uphoff Wasowski, John Campion, James Mathisen, Hillel Goldstein, Maria Bergman, Christine Gagnon, and Mary Anne Kubiak. 2000. "Stigmatizing Attributions about Mental Illness.” Journal of Community Psychology 28(1):91-102.

Corrigan, Patrick W., Amy C. Watson, Gabriela Gracia, Natalie Slopen, Kenneth Rasinski, and Laura L. Hall. 2005. "Newspaper Stories as Measures of Structural Stigma." Psychiatric Services 56(5):551-56.

Corrigan, Patrick W., Amy C. Watson, Mark L. Heyrman, Amy Warpinski, Gabriela Gracia, Natalie Slopen, and Laura L. Hall. 2005. "Structural Stigma in State Legislation." Psychiatric Services 56(5):557-63.

Crocetti, Guido M. 1974. Contemporary Attitudes toward Mental Illness. Pittsburgh: University of Pittsburgh Press.

Davies, Mark. 2012. "Expanding Horizons in Historical Linguistics with the 400-Million Word Corpus of Historical American English.” Corpora 7(2):121-57.

Dietrich, Sandra, Dirk Heider, Herbert Matschinger, and Matthias C. Angermeyer. 2006. "Influence of Newspaper Reporting on Adolescents' Attitudes toward People with Mental Illness.” Social Psychiatry and Psychiatric Epidemiology 41(4):318-22.

DiMaggio, Paul, Manish Nag, and David Blei. 2013. "Exploiting Affinities between Topic Modeling and the Sociological Perspective on Culture: Application to Newspaper Coverage of U.S. Government Arts Funding." Poetics 41(6):570-606. doi: 10.1016/j.poetic.2013.08.004.

Dwyer, Ellen. 1992. “Stories of Epilepsy, 1880-1930.” Pp. 248-72 in Framing Disease: Studies in Cultural History, edited by C. E. Rosenberg and J. Golden. Rutgers University Press.

Eliasoph, Nina, and Paul Lichterman. 2003. "Culture in Interaction.” American Journal of Sociology 108(4):735-94.

Else-Quest, Nicole M., Noelle K. LoConte, Joan H. Schiller, and Janet Shibley Hyde. 2009. "Perceived Stigma, Self-Blame, and Adjustment among Lung, Breast and Prostate Cancer Patients." Psychology and Health 24(8):949-64.

Epstein, Steven. 1996. Impure Science: AIDS, Activism, and the Politics of Knowledge. Berkeley, CA: University of California Press.

Estroff, Sue E., David L. Penn, and Julie R. Toporek. 2004. "From Stigma To Discrimination: An Analysis of Community Efforts To Reduce the Negative Consequences of Having a 
Psychiatric Disorder and Label." Schizophrenia Bulletin 30(3):493-509. doi: 10.1093/oxfordjournals.schbul.a007097.

Evans, John. 2009. “Two Worlds in Cultural Sociology.” Pp. 209-52 in Meaning and Method, edited by I. Reed and J. C. Alexander. Routledge.

Ferraro, Susan. 1993. "The Anguished Politics of Breast Cancer." The New York Times Magazine.

Fleischer, Doris Zames, and Frieda Zames. 2001. Disability Rights Movement: From Charity to Confrontation. Temple University Press.

Flint, Stuart W., Joanne Hudson, and David Lavallee. 2016. "The Portrayal of Obesity in UK National Newspapers." Stigma and Health 1(1):16.

Freidson, Eliot. 1970. Profession of Medicine: A Study of the Sociology of Applied Knowledge. University Of Chicago Press.

Garg, Nikhil, Londa Schiebinger, Dan Jurafsky, and James Zou. 2018. "Word Embeddings Quantify 100 Years of Gender and Ethnic Stereotypes." Proceedings of the National Academy of Sciences 115(16):E3635-44. doi: 10.1073/pnas.1720347115.

Giddens, Anthony. 1991. Modernity and Self-Identity: Self and Society in the Late Modern Age. Cambridge, U.K.: Polity Press.

Goffman, Erving. 1963. Stigma: Notes on the Management of Spoiled Identity. New York: Simon and Schuster.

Gollust, Sarah E., and Paula M. Lantz. 2009. "Communicating Population Health: Print News Media Coverage of Type 2 Diabetes." Social Science \& Medicine 69(7):1091-98. doi: 10.1016/j.socscimed.2009.07.009.

Gollust, Sarah E., Paula M. Lantz, and Peter A. Ubel. 2010. "Images of Illness: How Causal Claims and Racial Associations Influence Public Preferences toward Diabetes Research Spending." Journal of Health Politics, Policy and Law 35(6):921-59. doi: 10.1215/03616878-2010-034.

Gould, Deborah B. 2009. Moving Politics: Emotion and ACT UP's Fight against AIDS. University Of Chicago Press.

Gove, Walter R. 1982. "The Current Status of the Labeling Theory of Mental Illness." Deviance and Mental Illness 273-300.

Green, Gill. 2009. The End of Stigma? : Changes in the Social Experience of Long-Term Illness. Routledge.

Greene, Kathryn, and Smita C. Banerjee. 2006. "Disease-Related Stigma: Comparing Predictors of AIDS and Cancer Stigma." Journal of Homosexuality 50(4):185-209. 
Gunkel, Patrick. n.d. 638 Primary Personality Traits. Retrieved January 12, 2022 (http://ideonomy.mit.edu/essays/traits.html).

Haidt, Jonathan, and Craig Joseph. 2004. "Intuitive Ethics: How Innately Prepared Intuitions Generate Culturally Variable Virtues.” Daedalus 133(4):55-66.

Hatzenbuehler, Mark L., Anna Bellatorre, Yeonjin Lee, Brian K. Finch, Peter Muennig, and Kevin Fiscella. 2014. "RETRACTED: Structural Stigma and All-Cause Mortality in Sexual Minority Populations." Social Science \& Medicine 103:33-41. doi: 10.1016/j.socscimed.2013.06.005.

Hatzenbuehler, Mark L., and Bruce G. Link. 2014. "Introduction to the Special Issue on Structural Stigma and Health." Social Science \& Medicine 103:1-6. doi:

10.1016/j.socscimed.2013.12.017.

Hatzenbuehler, Mark L., Jo C. Phelan, and Bruce G. Link. 2013. "Stigma as a Fundamental Cause of Population Health Inequalities." American Journal of Public Health 103(5):813-21. doi: 10.2105/AJPH.2012.301069.

Herek, Gregory M. 2004. "Beyond 'Homophobia': Thinking about Sexual Prejudice and Stigma in the Twenty-First Century." Sexuality Research \& Social Policy 1(2):6-24.

Herek, Gregory M., John P. Capitanio, and Keith F. Widaman. 2002. "HIV-Related Stigma and Knowledge in the United States: Prevalence and Trends, 1991-1999." American Journal of Public Health 92(3):371-77. doi: 10.2105/AJPH.92.3.371.

Heuer, Chelsea A., Kimberly J. McClure, and Rebecca M. Puhl. 2011. "Obesity Stigma in Online News: A Visual Content Analysis.” Journal of Health Communication 16(9):97687.

Hoffman, Mark Anthony, Jean-Philippe Cointet, Philipp Brandt, Newton Key, and Peter Bearman. 2018. "The (Protestant) Bible, the (Printed) Sermon, and the Word(s): The Semantic Structure of the Conformist and Dissenting Bible, 1660-1780." Poetics 68:89103. doi: 10.1016/j.poetic.2017.11.002.

infoplease.com. 2007. "Top 100 Newspapers in the United States." InfoPlease. Retrieved December 19, 2019 (https://www.infoplease.com/arts-entertainment/newspapers-andmagazines/top-100-newspapers-united-states).

Janmaat, Jan G., and Avril Keating. 2019. “Are Today’s Youth More Tolerant? Trends in Tolerance among Young People in Britain." Ethnicities 19(1):44-65. doi: $10.1177 / 1468796817723682$.

Jepperson, Ronald L., and Ann Swidler. 1994. "What Properties of Culture Should We Measure?" Poetics 22(4):359-71. doi: 10.1016/0304-422X(94)90014-0. 
Jones, Edward E., Amerigo Farina, Albert H. Hastorf, Hazel Markus, Dale T. Miller, and Robert A. Scott. 1984. Social Stigma: The Psychology of Marked Relationships. New York: W H Freeman \& Co.

Joseph, Adam J., Neeraj Tandon, Lawrence H. Yang, Ken Duckworth, John Torous, Larry J. Seidman, and Matcheri S. Keshavan. 2015. "\# Schizophrenia: Use and Misuse on Twitter." Schizophrenia Research 165(2-3):111-15.

Joseph, Kenneth, and Jonathan Morgan. 2020. When Do Word Embeddings Accurately Reflect Surveys on Our Beliefs About People?

Kayal, Philip M. 1993. Bearing Witness: Gay Men's Health Crisis and the Politics of AIDS. Boulder: Westview Press.

Kedrowski, Karen M., and Marilyn S. Sarow. 2007. Cancer Activism: Gender, Media, and Public Policy. Chicago, IL: University of Illinois Press.

Kelly, John F., Richard Saitz, and Sarah Wakeman. 2016. "Language, Substance Use Disorders, and Policy: The Need to Reach Consensus on an "Addiction-Ary." Alcoholism Treatment Quarterly 34(1):116-23.

Kempner, Joanna. 2014. Not Tonight: Migraine and the Politics of Gender and Health. Chicago ; London: University Of Chicago Press.

King, Samantha. 2004. "Pink Ribbons Inc: Breast Cancer Activism and the Politics of Philanthropy." International Journal of Qualitative Studies in Education 17(4):473-92. doi: 10.1080/09518390410001709553.

Kool, Marianne B., Henriët van Middendorp, Mark A. Lumley, Johannes WJ Bijlsma, and Rinie Geenen. 2013. "Social Support and Invalidation by Others Contribute Uniquely to the Understanding of Physical and Mental Health of Patients with Rheumatic Diseases." Journal of Health Psychology 18(1):86-95.

Kozlowski, Austin C., Matt Taddy, and James A. Evans. 2019. "The Geometry of Culture: Analyzing the Meanings of Class through Word Embeddings." American Sociological Review 84(5):905-49.

Lebel, Sophie, and Gerald M. Devins. 2008. "Stigma in Cancer Patients Whose Behavior May Have Contributed to Their Disease." Future Oncology 4(5):717-33.

Lee, Monica, and John Levi Martin. 2015. "Coding, Counting and Cultural Cartography." American Journal of Cultural Sociology 3(1):1-33. doi: 10.1057/ajcs.2014.13.

Lerner, Barron H. 2001. The Breast Cancer Wars: Hope, Fear, and the Pursuit of a Cure in Twentieth-Century America. Oxford University Press, USA. 
Lesk, Michael. 1986. "Automatic Sense Disambiguation Using Machine Readable Dictionaries: How to Tell a Pine Cone from an Ice Cream Cone.” Pp. 24-26 in Proceedings of the 5th annual international conference on Systems documentation. ACM.

Link, B. G., J. C. Phelan, M. Bresnahan, A. Stueve, and B. A. Pescosolido. 1999. "Public Conceptions of Mental Illness: Labels, Causes, Dangerousness, and Social Distance." American Journal of Public Health 89(9):1328-33. doi: 10.2105/AJPH.89.9.1328.

Link, Bruce G., Francis T. Cullen, James Frank, and John F. Wozniak. 1987. “The Social Rejection of Former Mental Patients: Understanding Why Labels Matter." American Journal of Sociology 92(6):1461-1500.

Link, Bruce G., and Jo C. Phelan. 2001. "Conceptualizing Stigma.” Annual Review of Sociology $27: 363-85$.

Link, Bruce G., Jo C. Phelan, Richard Miech, and Emily Leckman Westin. 2008. “The Resources That Matter: Fundamental Social Causes of Health Disparities and the Challenge of Intelligence." Journal of Health and Social Behavior 49(1):72-91. doi: $10.1177 / 002214650804900106$.

Link, Bruce G., Lawrence H. Yang, Jo C. Phelan, and Pamela Y. Collins. 2004. "Measuring Mental Illness Stigma.” Schizophrenia Bulletin 30(3):511-41.

Lizardo, Omar. 2017. "Improving Cultural Analysis: Considering Personal Culture in Its Declarative and Nondeclarative Modes." American Sociological Review 82(1):88-115. doi: $10.1177 / 0003122416675175$.

Looper, Karl J., and Laurence J. Kirmayer. 2004. "Perceived Stigma in Functional Somatic Syndromes and Comparable Medical Conditions." Journal of Psychosomatic Research 57(4):373-78. doi: 10.1016/j.jpsychores.2004.03.005.

Lukachko, Alicia, Mark L. Hatzenbuehler, and Katherine M. Keyes. 2014. "Structural Racism and Myocardial Infarction in the United States." Social Science \& Medicine 103:42-50. doi: 10.1016/j.socscimed.2013.07.021.

Major, Brenda, and Laurie T. O'Brien. 2005. "The Social Psychology of Stigma." Annual Review of Psychology 56(1):393-421. doi: 10.1146/annurev.psych.56.091103.070137.

Mak, Winnie WS, Phoenix KH Mo, Rebecca YM Cheung, Jean Woo, Fanny M. Cheung, and Dominic Lee. 2006. "Comparative Stigma of HIV/AIDS, SARS, and Tuberculosis in Hong Kong.” Social Science \& Medicine 63(7):1912-22.

Marlow, Laura A. V., Jo Waller, and Jane Wardle. 2015. "Does Lung Cancer Attract Greater Stigma than Other Cancer Types?" Lung Cancer 88(1):104-7. doi: 10.1016/j.lungcan.2015.01.024.

McKinlay, J. B., and L. D. Marceau. 2002. "The End of the Golden Age of Doctoring." International Journal of Health Services 32(2):379-416. 
Metzl, Jonathan, and Anna Kirkland, eds. 2010. Against Health: How Health Became the New Morality. New York: NYU Press.

Mikolov, Tomas, Kai Chen, Greg Corrado, and Jeffrey Dean. 2013. "Efficient Estimation of Word Representations in Vector Space." ArXiv Preprint ArXiv:1301.3781.

Mikolov, Tomas, Ilya Sutskever, Kai Chen, Greg S. Corrado, and Jeff Dean. 2013. "Distributed Representations of Words and Phrases and Their Compositionality." Pp. 3111-19 in Advances in neural information processing systems.

Mintzes, Barbara, and Catherine Hodgkin. 1996. "The Consumer Movement: From Single-Issue Campaigns to Long-Term Reform." Pp. 76-91 in Contested ground: public purpose and private interest in the regulation of prescription drugs, edited by P. Davis. New York: Oxford University Press.

Mohr, John W. 1998. "Measuring Meaning Structures.” Annual Review of Sociology 24(1):34570. doi: 10.1146/annurev.soc.24.1.345.

Mohr, John W., Christopher A. Bail, Margaret Frye, Jennifer C. Lena, Omar Lizardo, Terence E. McDonnell, Ann Mische, Iddo Tavory, and Frederick F. Wherry. 2020. Measuring Culture. Columbia University Press.

Mohr, John W., and Petko Bogdanov. 2013. Introduction-Topic Models: What They Are and Why They Matter. Elsevier.

Morgen, Sandra. 2002. Into Our Own Hands: The Women's Health Movement in the United States, 1969-1990. Rutgers University Press.

Mukherjee, Siddhartha. 2010. The Emperor of All Maladies: A Biography of Cancer. New York: Scribner.

Nelson, Laura K. 2017. "Computational Grounded Theory: A Methodological Framework." Sociological Methods \& Research 0049124117729703. doi: $10.1177 / 0049124117729703$.

Nelson, Laura K. 2021. "Leveraging the Alignment between Machine Learning and Intersectionality: Using Word Embeddings to Measure Intersectional Experiences of the Nineteenth Century US South." Poetics 101539.

Oliver, Michael. 1990. The Politics of Disablement: A Sociological Approach. Palgrave Macmillan.

Pachankis, John E., Mark L. Hatzenbuehler, Katie Wang, Charles L. Burton, Forrest W. Crawford, Jo C. Phelan, and Bruce G. Link. 2018. "The Burden of Stigma on Health and Well-Being: A Taxonomy of Concealment, Course, Disruptiveness, Aesthetics, Origin, and Peril Across 93 Stigmas." Personality and Social Psychology Bulletin 44(4):451-74. doi: $10.1177 / 0146167217741313$. 
Pachucki, Mark A., and Ronald L. Breiger. 2010. "Cultural Holes: Beyond Relationality in Social Networks and Culture." Annual Review of Sociology 36(1):205-24. doi: 10.1146/annurev.soc.012809.102615.

Park, Kristin. 2002. “Stigma Management among the Voluntarily Childless.” Sociological Perspectives 45(1):21-45.

Parker, Richard, and Peter Aggleton. 2003. "HIV and AIDS-Related Stigma and Discrimination: A Conceptual Framework and Implications for Action." Social Science \& Medicine 57(1):13-24. doi: 10.1016/S0277-9536(02)00304-0.

Patterson, Amanda. 2014. Writers Write. Retrieved December 1, 2019 (https:/www.writerswrite.co.za/a-fabulous-resource-for-writers-350-character-traits/).

Patterson, Orlando. 2014. "Making Sense of Culture.” Annual Review of Sociology 40(1):1-30. doi: 10.1146/annurev-soc-071913-043123.

Payton, Andrew R., and Peggy A. Thoits. 2011. "Medicalization, Direct-to-Consumer Advertising, and Mental Illness Stigma." Society and Mental Health 1(1):55-70.

Pennington, Jeffrey, Richard Socher, and Christopher Manning. 2014. "Glove: Global Vectors for Word Representation." Proceedings of the 2014 Conference on Empirical Methods in Natural Language Processing (EMNLP).

Pescosolido, Bernice A. 2013. "The Public Stigma of Mental Illness: What Do We Think; What Do We Know; What Can We Prove?" Journal of Health and Social Behavior 54(1):1-21.

Pescosolido, Bernice A., and Jack K. Martin. 2015. "The Stigma Complex.” Annual Review of Sociology 41:87-116.

Pescosolido, Bernice A., Jack K. Martin, J. Scott Long, Tait R. Medina, Jo C. Phelan, and Bruce G. Link. 2010. “'A Disease like Any Other'? A Decade of Change in Public Reactions to Schizophrenia, Depression, and Alcohol Dependence." American Journal of Psychiatry 167(11):1321-30.

Phelan, Jo C. 2005. "Geneticization of Deviant Behavior and Consequences for Stigma: The Case of Mental Illness.” Journal of Health and Social Behavior 46(4):307-22.

Phelan, Jo C., and Bruce G. Link. 1998. “The Growing Belief That People with Mental Illnesses Are Violent: The Role of the Dangerousness Criterion for Civil Commitment." Social Psychiatry and Psychiatric Epidemiology 33(1):S7-12.

Phelan, Jo C., Bruce G. Link, Ann Stueve, and Bernice A. Pescosolido. 2000. "Public Conceptions of Mental Illness in 1950 and 1996: What Is Mental Illness and Is It to Be Feared?" Journal of Health and Social Behavior 41(2):188-207. doi: 10.2307/2676305. 
Rabinowitz, Joshua L., David O. Sears, Jim Sidanius, and Jon A. Krosnick. 2009. "Why Do White Americans Oppose Race-Targeted Policies? Clarifying the Impact of Symbolic Racism." Political Psychology 30(5):805-28.

Ray, Victor, and Louise Seamster. 2016. "Rethinking Racial Progress: A Response to Wimmer." Ethnic and Racial Studies 39(8):1361-69. doi: 10.1080/01419870.2016.1151540.

Read, J., N. Haslam, L. Sayce, and E. Davies. 2006. "Prejudice and Schizophrenia: A Review of the 'Mental Illness Is an Illness like Any Other' Approach." Acta Psychiatrica Scandinavica 114(5):303-18. doi: 10.1111/j.1600-0447.2006.00824.x.

Reavley, Nicola J., and Pamela D. Pilkington. 2014. "Use of Twitter to Monitor Attitudes toward Depression and Schizophrenia: An Exploratory Study.” PeerJ 2:e647.

Reed, Isaac Ariail. 2015. "Counting, Interpreting and Their Potential Interrelation in the Human Sciences." American Journal of Cultural Sociology 3(3):353-64. doi: 10.1057/ajcs.2015.7.

Řehůřrek, Radim, and Petr Sojka. 2010. "Software Framework for Topic Modelling with Large Corpora." Pp. 45-50 in Proceedings of the LREC 2010 Workshop on New Challenges for NLP Frameworks. ELRA.

Reutter, Linda I., Miriam J. Stewart, Gerry Veenstra, Rhonda Love, Dennis Raphael, and Edward Makwarimba. 2009. “'Who Do They Think We Are, Anyway?': Perceptions of and Responses to Poverty Stigma." Qualitative Health Research 19(3):297-311.

Robinson, Patrick, Daniel Turk, Sagar Jilka, and Matteo Cella. 2019. "Measuring Attitudes towards Mental Health Using Social Media: Investigating Stigma and Trivialisation." Social Psychiatry and Psychiatric Epidemiology; Heidelberg 54(1):51-58. doi: http://dx.doi.org/10.1007/s00127-018-1571-5.

Rodwin, Marc A. 1994. "Patient Accountability and Quality of Care: Lessons from Medical Consumerism and the Patients' Rights, Women's Health and Disability Rights Movements." American Journal of Law \& Medicine 20:147.

Roeloffs, Carol, Cathy Sherbourne, Jürgen Unützer, Arlene Fink, Lingqi Tang, and Kenneth B. Wells. 2003. "Stigma and Depression among Primary Care Patients." General Hospital Psychiatry 25(5):311-15.

Rong, Xin. 2014. "Word2vec Parameter Learning Explained." ArXiv Preprint ArXiv:1411.2738.

Rose, Nikolas. 2007. The Politics of Life Itself: Biomedicine, Power, and Subjectivity in the Twenty-First Century. Princeton University Press.

Rush, Ladonna L. 1998. "Affective Reactions to Multiple Social Stigmas." The Journal of Social Psychology 138(4):421-30. 
Ruzek, Sheryl Burt. 1979. The Women's Health Movement: Feminist Alternatives to Medical Control. Praeger Pub.

Saguy, Abigail C., and Kevin W. Riley. 2005. "Weighing Both Sides: Morality, Mortality, and Framing Contests over Obesity." Journal of Health Politics, Policy and Law 30(5):869923.

Sally E. Thorne, Carol Murray. 2000. "Social Constructions of Breast Cancer." Health Care for Women International 21(3):141-59. doi: 10.1080/073993300245221.

Sampson, Robert J., and Stephen W. Raudenbush. 2004. "Seeing Disorder: Neighborhood Stigma and the Social Construction of 'Broken Windows.'" Social Psychology Quarterly 67(4):319-42.

Sayce, Liz. 1998. "Stigma, Discrimination and Social Exclusion: What's in a Word?" Journal of Mental Health 7(4):331-43. doi: 10.1080/09638239817932.

Schneider, Anne, and Helen Ingram. 1993. "Social Construction of Target Populations: Implications for Politics and Policy." American Political Science Review 87(2):334-47.

Schneider, Joseph W., and Peter Conrad. 1980. "In the Closet with Illness: Epilepsy, Stigma Potential and Information Control*." Social Problems 28(1):32-44. doi: 10.2307/800379.

Shapiro, Joseph P. 1993. No Pity: People with Disabilities Forging a New Civil Rights Movement. New York: Times Books.

Sol, Mrs. 1993. "Character Traits.” Retrieved January 12, 2022 (http://classroomsol.weebly.com/uploads/1/1/2/0/1120439/character_traits.pdf).

Sontag, Susan. 1990. Illness as Metaphor ; and, AIDS and Its Metaphors. New York: Doubleday.

Spillman, Lyn. 2015. "Ghosts of Straw Men: A Reply to Lee and Martin." American Journal of Cultural Sociology 3(3):365-79. doi: 10.1057/ajcs.2015.5.

Star, Shirley A. 1952. What the Public Thinks about Mental Health and Mental Illness. National Opinion Research Center.

Starr, Paul. 1982. The Social Transformation of American Medicine: The Rise of a Sovereign Profession and the Making of a Vast Industry. Basic Books.

Steensland, Brian. 2010. "Moral Classification and Social Policy." Pp. 455-68 in Handbook of the Sociology of Morality, edited by S. Hitlin and S. Vaisey. New York: Springer.

Stipp, David. 1987. "Patrick Gunkel Is An Idea Man Who Thinks in Lists: And His Science of Ideonomy Has Intellectuals Dazzled, Well, Six Ways to Sunday Patrick Gunkel Has So Many Ideas He Must Keep Lists of His Lists - ProQuest.” The Wall Street Journal, June 1. 
Strauss, Claudia, and Naomi Quinn. 1997. A Cognitive Theory of Cultural Meaning. Vol. 9. Cambridge University Press.

Stuart, Heather L., Julio Arboleda-Flórez, and Norman Sartorius. 2012. Paradigms Lost: Fighting Stigma and the Lessons Learned. Oxford; New York : Oxford University Press.

Sullivan, Michael K. 2004. "Homophobia, History, and Homosexuality." Journal of Human Behavior in the Social Environment 8(2-3):1-13. doi: 10.1300/J137v08n02_01.

Swidler, Ann. 2013. Talk of Love: How Culture Matters. University of Chicago Press.

Tomes, Nancy. 2006. "The Patient as a Policy Factor: A Historical Case Study of the Consumer/Survivor Movement In Mental Health." Health Affairs 25(3):720-29.

Turner, Bryan S. 1992. Regulating Bodies: Essays in Medical Sociology. London, England; New York, N.Y: Routledge.

Vahabzadeh, Arshya, Justine Wittenauer, and Erika Carr. 2011. "Stigma, Schizophrenia and the Media: Exploring Changes in the Reporting of Schizophrenia in Major US Newspapers." Journal of Psychiatric Practice ${ }^{\circledR}$ 17(6):439-46.

Wailoo, Keith. 2006. "Stigma, Race, and Disease in 20th Century America." The Lancet 367(9509):531-33. doi: 10.1016/S0140-6736(06)68186-5.

Weiner, Bernard, Raymond P. Perry, and Jamie Magnusson. 1988. "An Attributional Analysis of Reactions to Stigmas." Journal of Personality and Social Psychology 55(5):738.

Wikipedia. 2019. "List of Newspapers in the United States." Wikipedia.

Williams, Rick L. 2000. "A Note on Robust Variance Estimation for Cluster-Correlated Data." Biometrics 56(2):645-46. doi: 10.1111/j.0006-341X.2000.00645.x.

Wood, Lisa, Michele Birtel, Sarah Alsawy, Melissa Pyle, and Anthony Morrison. 2014. "Public Perceptions of Stigma towards People with Schizophrenia, Depression, and Anxiety." Psychiatry Research 220(1-2):604-8.

“World Class Learning.” 2018. World Class Learning. Retrieved December 1, 2019 (https://www.worldclasslearning.com/english/character-trait-list.html).

Yahoo Finance. 2017. “America's 100 Largest Newspapers.” Retrieved December 19, 2019 (http://finance.yahoo.com/news/america-100-largest-newspapers-180528599.html).

Zola, Irving Kenneth. 1972. "Medicine as an Institution of Social Control." The Sociological Review 20(4):487-504. 


\section{Online Appendix: Methodological Details}

This appendix describes in detail how we cleaned text data from U.S. news articles across time, estimated a sequence of bootstrapped Word2Vec embedding models on this text data, and computed the stigmatizing meanings of our diseases across time using these embeddings (e.g.,

how the disease "depression" evokes danger, impurity, disgust, and negative personality traits). ${ }^{19}$

\section{Preprocessing Texts}

First, we combined multiple names for each disease into a single reconciled term. Most diseases can be referred to by multiple names. We compiled a list of terms for each disease using web searches and a literature review. To maximize the number of references to each disease Word2Vec could learn from, we used regular expressions in Python to search and replace all ways of mentioning the disease with a single, reconciled term.

In addition, some of our disease names had several potential meanings. For example, "alcoholic" may refer to a person or a drink; "MS" may abbreviate multiple sclerosis or Master of Science or be an honorific for a woman; and depression may refer to an illness or to a financial crisis. We disambiguated some polysemous terms by ensuring capitalization was consistent, such as "AIDS" rather than "aids." For other terms, we disambiguated words based on their context, using the Lesk algorithm in the NLTK package in Python (Lesk 1986). ${ }^{20}$ In some cases, we additionally explicitly pulled out closely related, non-disease terms (e.g., nonalcoholic, and MS-13) to further disambiguate these terms from references to diseases (e.g., alcoholic, and multiple sclerosis). We performed this reconciliation and disambiguation carefully

\footnotetext{
${ }^{20}$ After training a Word2Vec model on our cleaned corpus, we also checked the words with the highest cosine similarities to our ambiguous terms, to ensure that Word2 vec did indeed learn these terms as health conditions rather than other possible senses.
} 
and in pre-defined "rounds." This was important because certain references to diseases may be composed of other references (e.g., we first pulled out "social anxiety disorder" in a first round and then pulled out "anxiety disorder").

Next, we removed punctuation, and lowercased all data. We also converted two-word and three-word phrases into single terms, such that phrases like "New York" would be processed by Word2Vec as a single word, using a phraser model in Gensim (̌̌ehưřek and Sojka 2010). ${ }^{21}$

\section{Training Word Embeddings with Word2Vec}

To train our word embeddings with Word2Vec, we use the Context Bag of Words algorithm in the Python package Gensim (Řehůr̆ek and Sojka 2010). ${ }^{22}$ We set the context window at 10 words before and after each target word and the dimensionality of the wordvectors at 300, following previous work (Mikolov, Chen, et al. 2013; Mikolov, Sutskever, et al. 2013; Pennington, Socher, and Manning 2014; Rong 2014).

\section{Hyperparameter Selection}

We selected our hyperparameters for Word2Vec (dimensionality and context window) using a standard quality metric for word embeddings: we compare human-rated similarities between words with cosine-similarities based on Word2Vec. We use Spearman correlations for statistical comparison. The test set for human-rated similarities we use is WordSim353, which includes 353 similarities rated by at least 13 individuals (Finkelstein et al. 2002).

\footnotetext{
${ }^{21}$ For the hyperparameters for the phraser mode, we used a threshold of 12 and a minimum word count of 50 for each word or phrase.

${ }^{22}$ Word2Vec may use one of two training tasks: Context-Bag-of-Words (CBOW) or Skip-Gram. For CBOW, the algorithm's goal is to predict the target word-vector given the context word-vectors. Each time a mistake is made, the word-vectors are updated such that the correct answer would have been given. Across thousands and thousands of updates, the word-vectors become more and more meaningful. Skip-gram is very similar to CBOW; for this method, the algorithm's goal is to predict the context words given the target word.
} 
We first focused on selecting the optimal dimensionality. Using a single model trained on our final period with a context window of 5 , we trained models with dimensionality ranging from 100, 200, 300, and 400, and evaluated performance of each model on the WordSim353 Test. In the model with 100 dimensions, cosine similarities between words in this Word2Vec model had a Spearman correlation of 0.55 ( $\mathrm{p}$-value $<.0001)$ with human rated similarities between these same words. For 200, 300, and 400 dimensions, this correlation was $0.57,0.59$ and 0.59 (each pvalue $<.0001)$.

We then trained a model with dimensionality of 300 and now a wider context window (of 10 words, rather than 5) to tune the context window size. In this model cosine similarities between words in the model had a Spearman correlation of 0.62 (p-value $<.0001)$ with human rated similarities between these same words. This suggested that a context window of 10 resulted in higher quality models. Finally, to confirm that model quality was consistent across time periods, we examined the correlation between cosine similarities and human rated similarities in each time-period: on average, the Spearman correlation was $0.62(\mathrm{SD}=0.02$, each $\mathrm{p}$ value $<.0001$ ). Thus, our final dimensionality was set at 300 and we used a context window size of 10 .

In these models trained on all data, we used 5 iterations and a minimum word count of 50. In our final bootstrapped modeling (described next), we lowered the number of iterations to 3 , for reduce computation time. The minimum word count was still 50 .

\section{Training Bootstrapped Embeddings}

Upon selecting our hyperparameters, we trained bootstrapped embeddings on our data across time. Specifically, for each of the three-year time window of data, we trained 25 bootstrapped 
embeddings. Data for each bootstrapped embedding was created by randomly resampling articles in the time window (with replacement, to be the same size as the total number of articles in the time window). This process yielded 25 bootstrapped embeddings per time window, for a total of 325 embeddings across all 13-time periods.

\section{Post-Processing Certain Diseases in Bootstrapped Embeddings}

As described earlier, our initial list of diseases included a wide variety of ways to reference each disease, such as "obesity" and "obese." During our initial preprocessing of text data, we kept the adjective and noun forms for diseases separate for certain diseases where the adjective form might be especially stigmatizing compared to the noun form (e.g., reconciling one term for the various references to "obesity" and another for the various references to "obese"); this was the case for obese/obesity, epileptic/epilepsy, and drug addict/addiction. We collapsed both adjective and noun for other diseases where the adjective form was more neutral (e.g., all variants of "diabetic" and "diabetes" were collapsed into one term "diabetes"). After and training word embeddings on the cleaned data and beginning analyses, we realized that it may make more sense to have a single term for all adjective and noun forms (regardless of the disease). To fix this post-hoc, in each bootstrapped model we created a new word-vector: epilepsy_folded, drug_addiction_folded, and obesity_folded. Each word-vector was a weighted average of the word-vector noun and verb form, where weights adjust the contribution of the word-vector based on its frequency in the corpus (e.g., since "epilepsy" is more common in the corpus than "epileptic", the former gets relatively more weight than the latter in the weighted average).

We pilot tested our cleaning and reconciliation procedures and then trained word embeddings on New York Times Annotated Corpus news data. We then cleaned and reconciled 
our corpus collected using the LexisNexis API, trained word embeddings on this cleaned data, selected hyperparameters for Word2Vec training (this training was performed on all data in each time period, without bootstraps, described next), and made several adjustments to our cleaning code after working with these embeddings. Finally, we re-cleaned and processed our whole corpus again, now in a way that also enabled us to perform bootstrapping by article.

\section{Extracting and Validating Stigma Dimensions}

To extract a dimension, we first selected two sets of "anchor words," each of which represents a pole of the dimensions, such as purity and impurity. Second, using these anchor words we extracted a extracted a line corresponding to the dimension (e.g., impurity) in semantic space. Specifically, once our anchor words were finalized, we averaged the word-vectors for the first pole (e.g., impurity), averaged the word-vectors for the second pole (e.g., purity), and then subtracted these two averages. This yields a vector corresponding to our stigma's meaning (e.g., a line from pure to impure).

To measure the meaning of a new word or disease, like "schizophrenia," we computed the cosine similarity between the word-vector for "schizophrenia" and the stigma dimension. Conceptually, this corresponds to how the meaning of the word-vector for "schizophrenia" relates to the stigma dimensions. This cosine similarity yields a positive or negative "stigma score" ranging from -1.0 to $1.0,{ }^{23}$ where a more positive score suggests that "schizophrenia" lies closer to the stigmatized pole of the dimension (e.g., impurity), and a more negative score suggests that "schizophrenia" lies closer to the non-stigmatized pole of the dimension (e.g., purity). A cosine similarity of 0 suggests that the word is not used in a way that systematically

\footnotetext{
${ }^{23}$ We normalize word-vectors and stigma dimensions to be unit-length prior to analyses.
} 
corresponds to either end of the dimension. Note the sign of the score indicates which pole a term (e.g., "schizophrenia") lies closer too, and the magnitude of the score indicates how close the term lies to the pole. We code all stigma dimensions such that positive scores indicate more stigma.

To render scores more interpretable, we then standardize the score against the scores for all other vocabulary words in the embedding. Now, a score is the number of standard deviations more (or less) stigmatized a word is compared to all other vocabulary words in the embedding. This approach also accounts of potential differences in overall way that a stigma dimension is used across times, such as if dangerousness were de-emphasized in all news language (not only disease terms) across time. We apply this overall method to measure the extent to which each of our diseases are represented in our data as dangerous, impure, disgusting, and associated with negative personality traits.

\section{Selecting Anchor Words}

We began with a set of anchor word-vectors to represent each end of the dimension (e.g., words that represent purity and words that represent impurity, to create the impurity dimension). To select these anchor words, we used established lexicons from Moral Foundations Theory for impurity (Haidt and Joseph 2004). For disgust and danger, we developed word lists using a thesaurus (see Table A1 for sample words from each list). ${ }^{24}$

\section{TABLE A1 ABOUT HERE}

To select a set of anchor words for personality traits, we gathered words from a variety of online sources of word lists intended as resources for writers, from a variety of time periods: 1) a list of positive and negative traits which were described in a 1987 Wall Street Journal article

\footnotetext{
${ }^{24}$ Complete word lists are available from the authors upon request.
} 
(Gunkel n.d.; Stipp 1987); 2) contemporary list of positive and negative traits for fiction writers (A. Patterson 2014); 3) a word list if character traits designed as a resource for English language learners ("World Class Learning" 2018); 4) a handout of character traits for classrooms (Sol 1993). Only anchor words prevalent at least 50 times in an embedding were used to compute a dimension in a given embedding.

\section{Validating Dimension Quality}

Next, we used cross validation to test how accurately our dimensions classified the anchor words (e.g., as disgusting versus enticing). As described above, our "stigma scores" may be interpreted as continuous or categorical (using the sign or magnitude); for classification we use the sign of the score to indicate which pole the term is classified as closest to. Specifically, using a given embedding, we extracted each dimension using $90 \%$ of the anchor words and then tested how accurately our extracted dimension classified (based on the sign of the stigma score) the remaining $10 \%$ of anchor words ("hold-out set). We repeated this across all possible sets of $90 \%$ of anchor words, and compute the average accuracy on the hold-out sets. Performance on held out sets are listed in table A2.

\section{TABLE A2 ABOUT HERE}

For our four dimensions, the average accuracy across held-out sets across all years (for the first bootstrapped model) ranged from $89 \%$ to $95 \%$, suggesting that each of our stigma dimensions can successfully distinguish their corresponding relational concepts (e.g., the difference between disgusting and enticing) (see Table A2).

To check the face validity of our dimensions, we examined the words in the vocabulary that load highest onto each end of each dimension. For all dimensions, the words make substantive sense, 
adding to our confidence that the dimensions capture the stigma concepts (see Table A3). ${ }^{25}$

\section{TABLE A3 ABOUT HERE}

Finally, we compare our stigma measures to Pachankis et al.'s (2018) survey of 64 scholars who had published highly cited articles about stigma. These experts were asked to rate several types of stigma targeting several dozen conditions, including 21 health conditions in our study and 35 additional conditions appearing in our Word2Vec models.

Social distance for each stigmatized condition was measured using a seven-item Social Distance Scale (Link et al. 1987). This scale assesses how willing individuals are to interact with people from a given stigmatized status, across various contexts (e.g., how willing they would be to work with, or be a neighbor of the person with the stigmatized status). Experts rated the items between 0 (definitely unwilling) to 3 (definitely willing). They were asked to respond "in terms of their understanding of general social perception." Following Pachankis et al. (2018), we derived an index for social distance by computing the mean across all seven items. As reported by Pachankis et al., the “average Cronbach's alpha for social distance across stigmatized statuses was 0.84 for the expert raters."

These expert responses of social distance for stigmatizing conditions were correlated with our measures of disgust, impurity, danger, and negative-positive traits with Spearman correlation coefficients of $0.36(p<.01), 0.60(p<.0001), 0.29(p<.05)$, and $0.53(p<.0001)$, respectively. ${ }^{26}$ Furthermore, the expert responses are correlated with our stigma index with a Spearman correlation of $0.59(\mathrm{p}<.0001)$. Using the less conservative Pearson correlations coefficient we find similar conclusions, with slightly higher correlation coefficients: $0.36(p<.01), 0.63$ $(\mathrm{p}<.0001), 0.37(\mathrm{p}<.01)$, and $0.50(\mathrm{p}<.001)$.

\footnotetext{
25 The somewhat obscure words identified as the most pure all refer to religious institutions and concepts.

${ }^{26}$ For comparability to Pachankis et al., (2018) we use our text-based measures from the 2013-2015 time window, which most closely corresponds to when Pachankis and colleagues collected their survey data.
} 
To measure disgust in Pachankis et al., the experts were asked "to what extent does the condition prompt physical revulsion among typical members of the U.S. population in typical social interactions, assuming the stigma is known?" (Pachankis et al. 2018:456). The expert responses were correlated with our measure of disgust, with a Spearman correlation coefficient of $0.45(p<.0001)$, and Pearson correlation coefficient of $0.43(p<.001)$.

Turning to danger, Pachankis et al. asked the experts, "In the general U.S. population, to what extent do people who interact with the stigmatized individual perceive some kind of contagion, threat, peril, or physical danger to themselves in typical social interactions, assuming the stigma is known?" (Pachankis et al. 2018:456). There are two key differences between this survey measure and our danger dimension. The survey question explicitly includes the threat of contagion, while we excluded direct references to contagion from the word list we used to construct the danger dimension. ${ }^{27}$ The survey also explicitly focused attention on danger to those interacting with a patient, while the danger picked up by Word2Vec could include dangers to the patients themselves. Perhaps because of these differences, our text-based measures of danger diverged dramatically from human coding, with a Spearman correlation coefficient of only 0.23 $(p=.09)$ and Pearson correlation coefficient of $0.32(p<.05)$. Figure A.1 shows our text-based measures of danger on the x-axis, and expert coding on the y-axis. There was some overlap in the human and text-based coding-for example, both methods rated schizophrenia as dangerous and mental retardation as safe. But drug addiction, HIV, and herpes were coded as much more threatening by human coders than by text. So was psoriasis, which coders predicted would be moderately threatening, but was not constructed as dangerous in text.

FIGURE A1 ABOUT HERE 


\section{FIGURE A2 ABOUT HERE}

Text-based and human-coded measures of morality show more congruence, with a Spearman correlation coefficient of $0.57(\mathrm{p}<.0001)$ and Pearson correlation coefficient of 0.62 $(\mathrm{p}<.0001)$ (see Figure A.2). Pachankis and colleagues asked their expert respondents, "To what extent do typical members of the U.S. population see the stigmatized person as having a moral blemish associated with individual failings?" ${ }^{28}$ Most of the diseases cluster around an upwardsloping line, suggesting that diseases that humans coded as more immoral were also constructed this way in text. One interesting divergence was that the experts predicted drug addiction, alcoholism, and HIV infection to be highly associated with immorality. However, these conditions do not seem to be constructed as immoral in the contemporary news media, perhaps because they have been so successfully medicalized. ${ }^{29}$ 


\section{Tables and Figures (Main Text)}

Table 1: News Media Sources

The Atlanta Journal-Constitution, The Austin American-Statesman, The Daily Oklahoman, The Mercury News (California), The Minneapolis Star

Newspapers Tribune, The New York Post, The New York Times, The Philadelphia Inquirer, Pittsburgh Post-Gazette, Richmond Times Dispatch, The San Diego Union Tribune, St. Louis Post-Dispatch, St. Paul Pioneer Press, Sun-Sentinel (Fort Lauderdale), Tampa Bay Times, The Washington Post, USA Today

News Wires $\quad$ Associated Press, Reuters

News Magazines Newsweek, U.S. News \& World Report

TV and Radio ABC News, CBS News, Fox News Network, MSNBC, NBC News, NPR All Things Considered 
Table 2: Sampled Diseases by Category

Mental Illnesses

Addictions

Eating Disorders

Sexually Transmitted Infections

Other Infectious Diseases

Lifestyle Causes

Cancers

Contested Illnesses

Autoimmune

Genetic

Musculoskeletal

Neurodevelopmental

Neurological

Visual / Auditory Impairments

Other
Anxiety Disorder, Bipolar Disorder, Borderline Disorder, Depression, Dissociative Disorder, Obsessive Compulsive Disorder, PTSD, Schizophrenia, Social Anxiety Disorder Alcoholism, Drug Addiction

Anorexia, Bulimia

Chlamydia, Gonorrhea, Herpes, HIV / AIDS, Human

Papillomavirus, Syphilis

Hepatitis, Influenza, Lyme Disease, Malaria, Pelvic

Inflammatory Disease, Pneumonia, Septicemia, Tuberculosis

Atherosclerosis, Cirrhosis, COPD, Diabetes, Heart Disease, Hypertension, Kidney Disease, Obesity, Stroke Brain Cancer, Breast Cancer, Cervical Cancer, Colon Cancer, Leukemia, Liver Cancer, Lung Cancer, Lymphoma, Myeloma, Ovarian Cancer, Pancreatic Cancer, Prostate Cancer, Skin Cancer, Uterine Cancer

Chronic Fatigue Syndrome, Fibromyalgia, Gulf War Syndrome, Inflammatory Bowel Disease, Multiple Chemical Sensitivity

Asthma, Celiac Disease, Eczema, Lupus, Psoriasis, Scleroderma

Alpha One, Cystic Fibrosis, Epidermolysis Bullosa, Fragile $\mathrm{X}$, Hemochromatosis, Hemophilia, Huntington's Disease, Marfan Syndrome, Neurofibromatosis, Osteogenesis Imperfecta, Phenylketonuria, Prader Willi Syndrome, Rett Syndrome, Sickle Cell Anemia, Spinal Muscular Atrophy, Tay Sachs, Thalassemia, Tuberous Sclerosis Arthritis, Osteoporosis, Pagets Disease, Scoliosis ADHD, Autism, Down Syndrome, Dyslexia, Tourette Syndrome

ALS, Alzheimer's, Cerebral Palsy, Epilepsy, Headache, Multiple Sclerosis, Muscular Dystrophy, Myasthenia Gravis, Parkinsons, Spina Bifida

Glaucoma, Hearing Impairment, Visual Impairment Cleft Palate, Hydrocephalus, Interstitial Cystitis, Restless Leg Syndrome, Reye Syndrome 
Table 3: Descriptive Statistics

\begin{tabular}{lrrrr}
\hline & Mean & SD & Min & Max \\
\hline Stigma index & 0.80 & 0.66 & -0.76 & 2.22 \\
Disgust score & 1.13 & 0.74 & -0.95 & 3.07 \\
Danger score & 0.84 & 0.74 & -1.46 & 2.91 \\
Impurity score & 0.59 & 0.70 & -1.18 & 2.49 \\
Negative traits score & 0.63 & 0.91 & -1.40 & 3.01 \\
Media mentions & 0.00 & 0.06 & -0.04 & 0.66 \\
Medicalization & 3.35 & 0.79 & -0.64 & 5.47 \\
Advocacy index & 0.05 & 0.98 & -0.38 & 11.71 \\
\hline
\end{tabular}

Note: Data are pooled across all time periods 
Table 4: Predicting Disease Stigma
(1)
(2)
(3)
(4)
(5)

\begin{tabular}{|c|c|c|c|c|c|}
\hline Year $(1980=1)$ & $\begin{array}{c}-0.059 * * * \\
(0.006)\end{array}$ & $\begin{array}{c}-0.059 * * * \\
(0.006)\end{array}$ & $\begin{array}{c}-0.067 * * * \\
(0.006)\end{array}$ & $\begin{array}{c}-0.062 * * * \\
(0.006)\end{array}$ & $\begin{array}{c}-0.050 * * * \\
(0.008)\end{array}$ \\
\hline Mental Illness & & $\begin{array}{c}0.877 * * * \\
(0.071)\end{array}$ & $\begin{array}{c}0.478^{* * *} \\
(0.122)\end{array}$ & $\begin{array}{c}0.530 * * * \\
(0.138)\end{array}$ & $\begin{array}{c}0.307 \\
(0.174)\end{array}$ \\
\hline Mental Illness x Year & & & $\begin{array}{c}0.051 * * * \\
(0.012)\end{array}$ & $\begin{array}{c}0.048 * * * \\
(0.011)\end{array}$ & $\begin{array}{c}0.076^{* * *} \\
(0.014)\end{array}$ \\
\hline Eating Disorder & & $\begin{array}{c}1.087 * * * \\
(0.058)\end{array}$ & $\begin{array}{c}0.574 * * * \\
(0.078)\end{array}$ & $\begin{array}{c}0.528 * * * \\
(0.075)\end{array}$ & $\begin{array}{c}0.994 * * * \\
(0.082)\end{array}$ \\
\hline Eating Disorder x Year & & & $\begin{array}{c}0.073 * * * \\
(0.006)\end{array}$ & $\begin{array}{c}0.084 * * * \\
(0.008)\end{array}$ & $\begin{array}{c}0.049 * * * \\
(0.008)\end{array}$ \\
\hline Addiction & & $\begin{array}{c}0.891 * * * \\
(0.066)\end{array}$ & $\begin{array}{c}0.244 * * \\
(0.079)\end{array}$ & $\begin{array}{c}0.453 * * * \\
(0.101)\end{array}$ & $\begin{array}{c}0.777 * * * \\
(0.157)\end{array}$ \\
\hline Addiction $\mathrm{x}$ Year & & & $\begin{array}{c}0.092 * * * \\
(0.010)\end{array}$ & $\begin{array}{c}0.093 * * * \\
(0.012)\end{array}$ & $\begin{array}{c}0.076 * * * \\
(0.011)\end{array}$ \\
\hline Media Mentions & & & & $\begin{array}{c}-1.930 * \\
(0.762)\end{array}$ & $\begin{array}{l}-0.195 \\
(0.771)\end{array}$ \\
\hline Medicalization & & & & $\begin{array}{c}0.164 * * * \\
(0.048)\end{array}$ & $\begin{array}{c}0.152 * * \\
(0.046)\end{array}$ \\
\hline Advocacy & & & & & $\begin{array}{c}-0.296 * * \\
(0.106)\end{array}$ \\
\hline Constant & $\begin{array}{c}1.237 * * * \\
(0.071)\end{array}$ & $\begin{array}{c}1.117 * * * \\
(0.075)\end{array}$ & $\begin{array}{c}1.181 * * * \\
(0.077)\end{array}$ & $\begin{array}{c}0.579 * * \\
(0.188)\end{array}$ & $\begin{array}{l}0.411^{*} \\
(0.177)\end{array}$ \\
\hline Observations & 1,101 & 1,101 & 1,101 & 1,101 & 734 \\
\hline $\mathrm{R}$-squared & 0.098 & 0.321 & 0.335 & 0.402 & 0.414 \\
\hline
\end{tabular}

Robust standard errors in parentheses $* * * \mathrm{p}<0.001, * * \mathrm{p}<0.01, * \mathrm{p}<0.05$ 


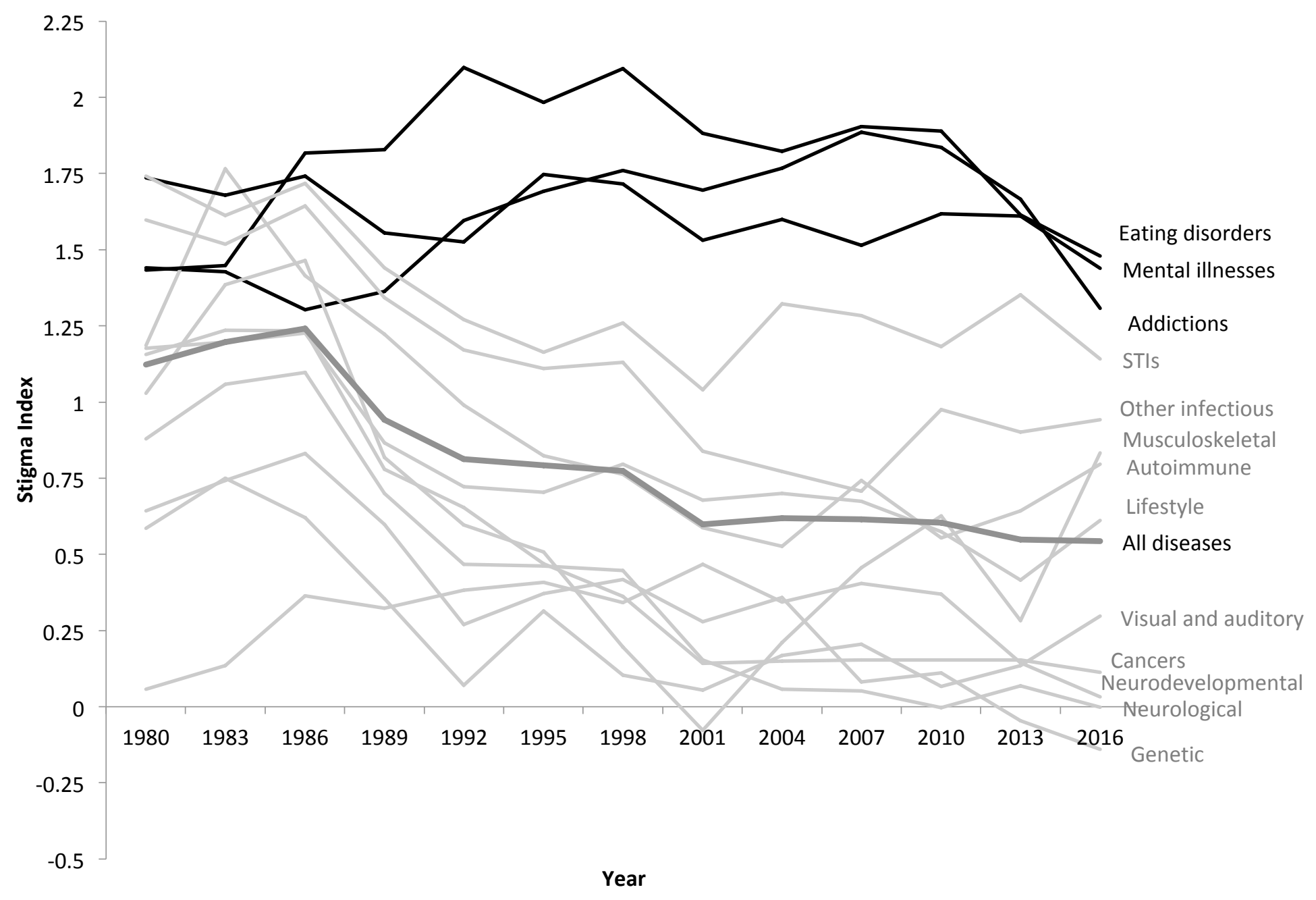

Figure 1: Uneven Stigma Declines Across Disease Categories 


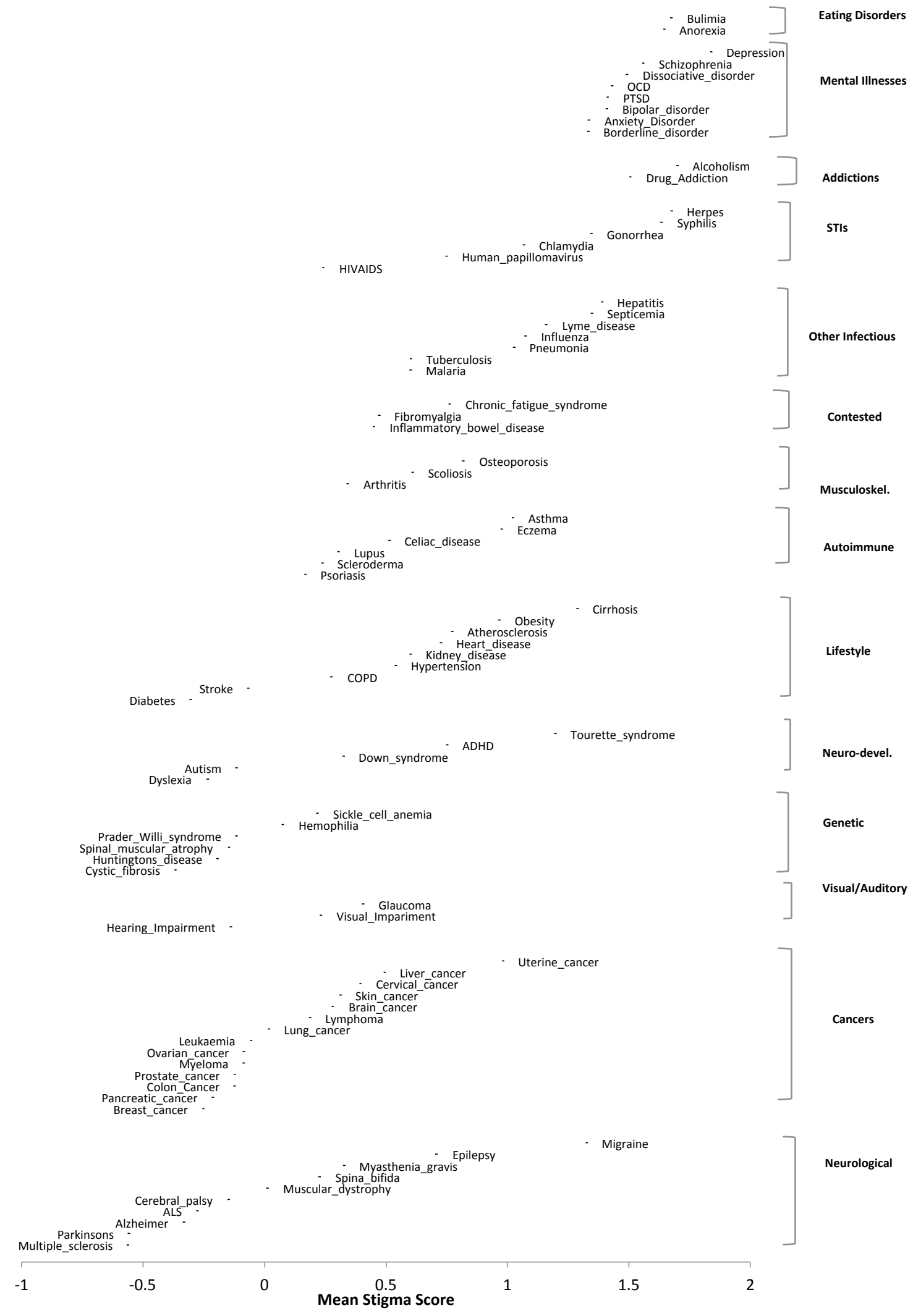

Figure 2: Mean stigma scores for diseases, 2010-2018 


\section{Tables and Figures (Methodological Appendix)}

Table A1: Sample Anchor Words for Stigma Dimensions

\begin{tabular}{lll}
\hline Stigma Dimension & Sample Anchor Words (Pole 1) & Sample Anchor Words (Pole 2) \\
\hline Danger & $\begin{array}{l}\text { Afraid, alarm, crisis, dangerous, } \\
\text { doom }\end{array}$ & $\begin{array}{l}\text { Assured, comforted, protect, refuge, } \\
\text { safe }\end{array}$ \\
Impurity & $\begin{array}{l}\text { Defile, depraved, indecent, } \\
\text { debase, obscene }\end{array}$ & $\begin{array}{l}\text { Wholesome, immaculate, piety, } \\
\text { pristine, pure }\end{array}$ \\
& $\begin{array}{l}\text { Decay, repulsive, deformed, } \\
\text { disgusting, gross }\end{array}$ & $\begin{array}{l}\text { Alluring, appealing, attractive, } \\
\text { captivating, enticing }\end{array}$ \\
Disgust & $\begin{array}{l}\text { Arrogant, pessimistic, careless, } \\
\text { Pegative }\end{array}$ & $\begin{array}{l}\text { Compassionate, adventurous, } \\
\text { charismatic, intelligent, loyal }\end{array}$ \\
\hline
\end{tabular}

Table A2: Cross-Validation Accuracy on Held Out Folds

\begin{tabular}{lllll}
\hline & Danger & Impurity & Disgust & Negative Traits \\
Mean N (SD) & $8(0.46)$ & $5(0.49)$ & $8(0.82)$ & $42(1.7)$ \\
Mean Percent (SD) & $92 \%(3.4 \%)$ & $89 \%(3.5 \%)$ & $95 \%(1.5 \%)$ & $91 \%(1.8 \%)$ \\
\hline
\end{tabular}

Note: Means are calculated across all time periods, using one bootstrapped embedding from each time period (the first bootstrap). 
Table A3: Most Similar and Least Similar Words to Stigma Dimensions

$\begin{array}{lll}\text { Dimension / Five most similar words } & \text { Five least similar words } \\ \text { Time Period } & \text { Fim }\end{array}$

Danger

2016-2018

$1998-2000$

1980-1982

Impurity

2016-2018

$1998-2000$

1980-1982

Disgust

2016-2018

$1998-2000$

1980-1982

Negative Traits

2016-2018

$1998-2000$

1980-1982
Frightening, terrifying, bizarre, horrifying, violent

Frightening, terrifying, bizarre, horrifying, violent

Violent, vicious, frightening, mayhem, political turmoil

Mishandled, harassing, smearing, perpetrated, retaliated against

Mishandling, mishandled, tainted, cover_ups, scandals_involving

Stung, aggravated, triggered, beatings, being_investigated

Excrement, stench, feces, rotting, sores

Feces, stench, rotting, mildew, residue

Poison_ivy, concussion, fumes, machete, dynamite

Unpatriotic, insensitive, misogynistic, grossly, uninformed

Bullying, temper_tantrums, humiliated, vile, cursing

Beatings, harassment, bungling, rapists, unfairly
Ensure, secure, protect, maintain, safeguard

Secure, ensuring, ensure, assure, safeguard

Obtain, protect, assuring, ensure, safeguard

Harmony sturdy, pastoral, rustic, continuity

Monastery, shepherds, harmony, pastoral, nondenominational

Self_reliance, shrine, cathedral, monastery, pastoral

Wowed, introduces, wowing, attracting, entice

Wowed, attracted, captivated, enticed, adventurous

Attracted, showcase, selected, adventurous, presenting

Collaborative, thoughtful, innovative, high_quality, world_class

Well_rounded, dedication, innovative, thoughtful, superb

Comprehensive, showcase, ensemble, prestigious, first_rate

Note: Uses one bootstrapped embedding from each time-period (the first bootstrap). Similarity measured using cosine similarity. 


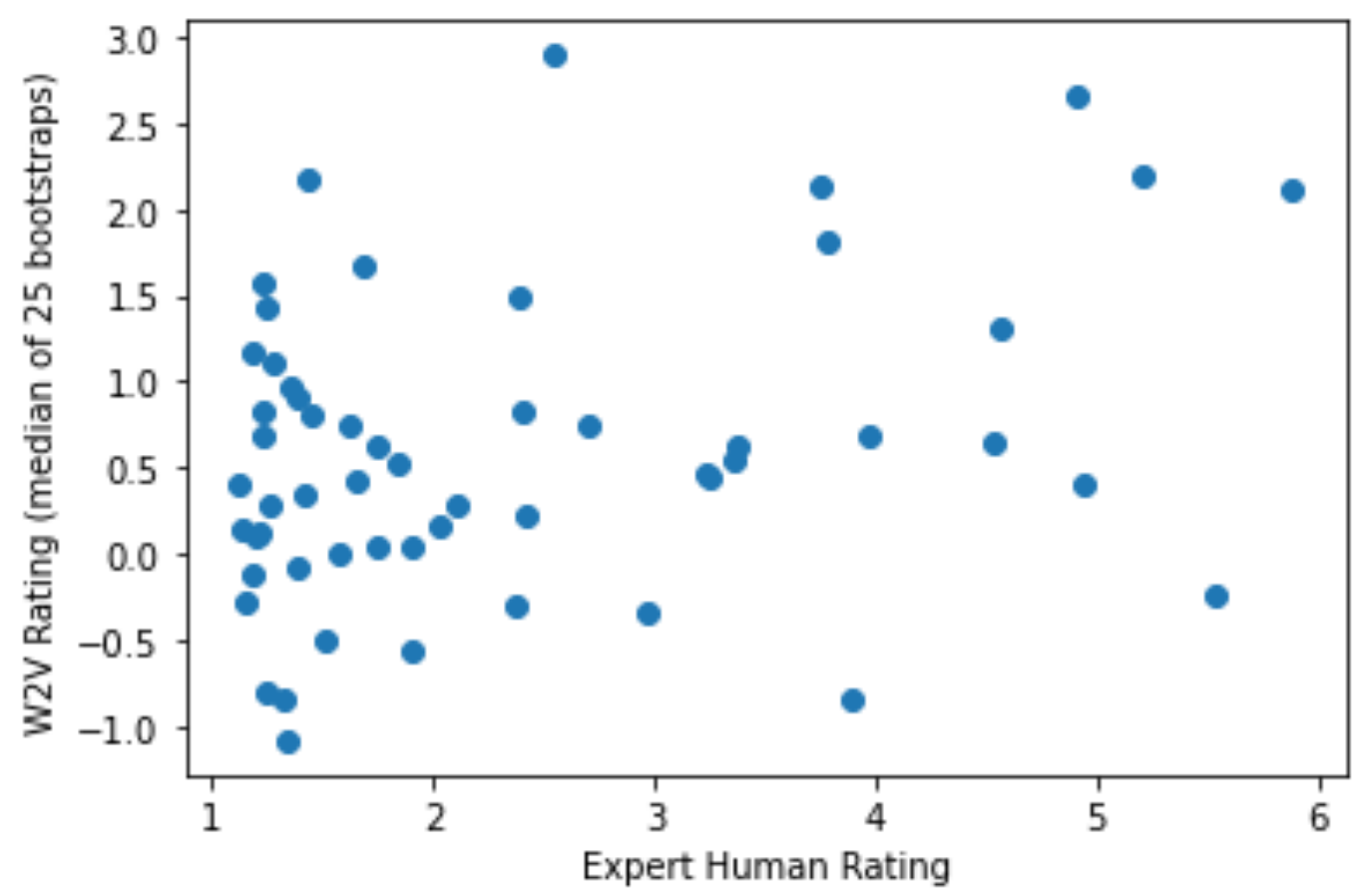

Figure A.1: Our text-based measures of danger on the y-axis (using Word2Vec), and expert coding on the x-axis (from survey data collected by Pachankis et. al. (2018)) for 56 stigmatized identities in Pachankis et al.

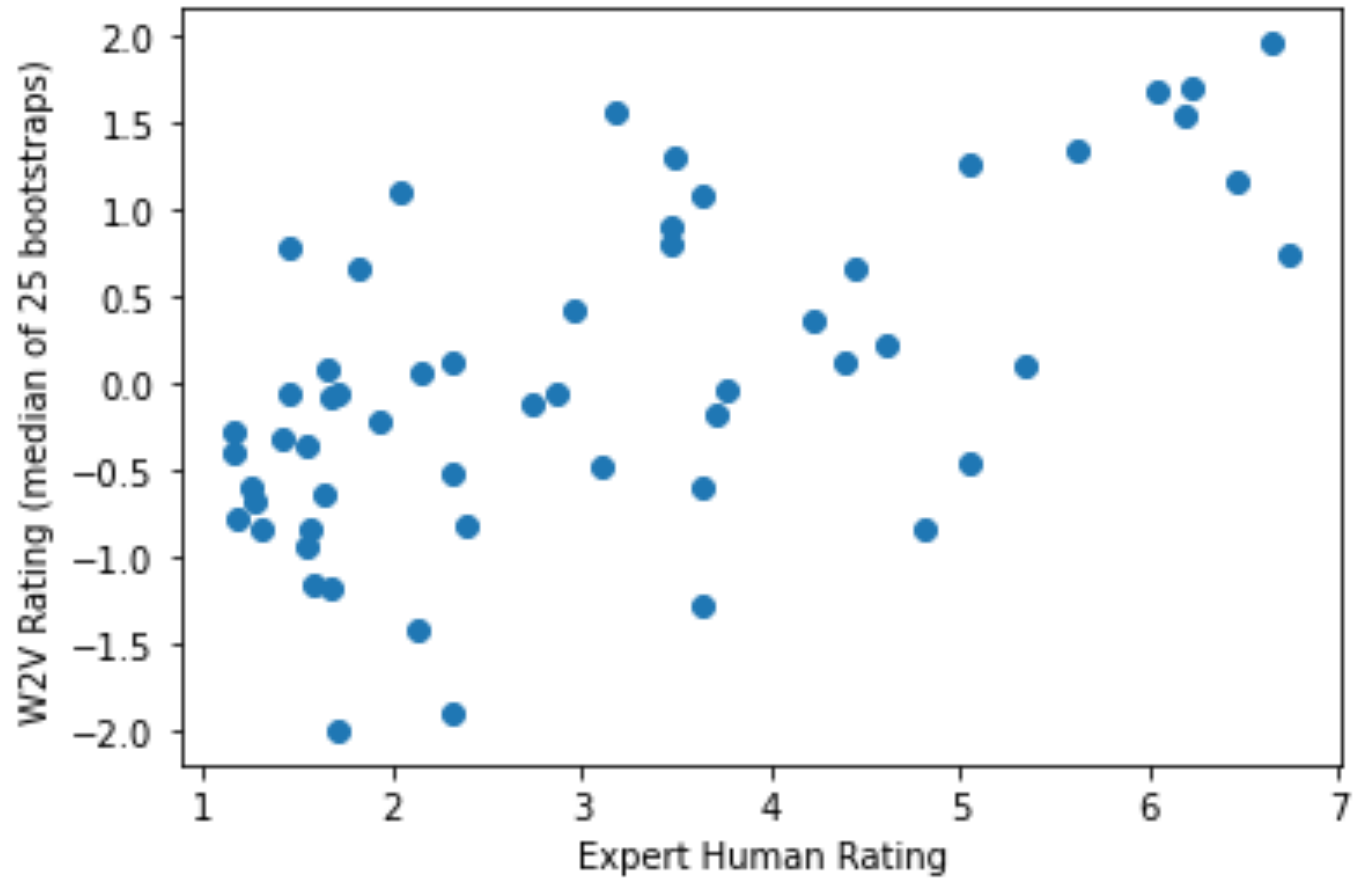

Figure A.2: Our text-based measures of impurity on the y-axis (using Word2Vec), and expert coding on the $\mathrm{x}$-axis (from survey data collected by Pachankis et. al. (2018)) for 56 stigmatized identities in Pachankis et al. (2018). 